\title{
Loss of glutamate transporter eaat $2 a$ leads to aberrant neuronal excitability, recurrent epileptic seizures and hypoactivity
}

\author{
Adriana L. Hotz ${ }^{1,2}$, Ahmed Jamali ${ }^{3}$, Nicolas N. Rieser ${ }^{1,2}$, Stephanie Niklaus ${ }^{1,7}$, Ecem Aydin ${ }^{3}$, Sverre \\ Myren-Svelstad ${ }^{3,4,5}$, Laetitia Lalla ${ }^{3}$, Nathalie Jurisch-Yaksi ${ }^{3,4,6, *}$, Emre Yaksi ${ }^{3{ }^{3,}}$ \& Stephan C.F. \\ Neuhauss ${ }^{1, *, 8}$
}

${ }^{1}$ Department of Molecular Life Sciences, University of Zurich, CH-8057 Zurich, Switzerland
${ }^{2}$ Life Science Zürich Graduate School, CH- 8057 Zurich, Switzerland
${ }^{3}$ Kavli Institute for Systems Neuroscience and Centre for Neural Computation, Faculty of Medicine and Health Sciences, Norwegian University
of Science and Technology, 7030 Trondheim, Norway
${ }^{4}$ Department of Neurology and Clinical Neurophysiology, St Olav University Hospital, 7030 Trondheim, Norway
${ }^{5}$ Department of Neuromedicine and Movement Science, Faculty of Medicine and Health Sciences, Norwegian University of Science and
Technology, 7030 Trondheim, Norway
${ }^{6}$ Department of Clinical and Molecular Medicine, Norwegian University of Science and Technology, 7030 Trondheim, Norway
${ }^{7}$ Current address: EraCal Therapeutics, CH-8952 Schlieren, Switzerland

*Corresponding authors: stephan.neuhauss@mls.uzh.ch, nathalie.jurisch-yaksi@,ntnu.no, emre.yaksi@ntnu.no

\begin{abstract}
Astroglial excitatory amino acid transporter 2 (EAAT2, GLT-1, SLC1A2) regulates the duration and extent of neuronal excitation by removing glutamate from the synaptic cleft. Hence, an impairment in EAAT2 function could lead to an imbalanced neural network excitability. Here, we investigated the functional alterations of neuronal and astroglial networks associated with the loss of function in the astroglia predominant eaat $2 a$ gene in zebrafish. We observed that eaat $2 a^{-/}$mutant zebrafish larvae display recurrent spontaneous and light-induced seizures in neurons and astroglia, which coincide with an abrupt increase in extracellular glutamate levels. In stark contrast to this hyperexcitability, basal neuronal and astroglial activity was surprisingly reduced in eaat $2 a^{-/-}$ mutant animals, which manifested in decreased overall locomotion. Our results reveal an essential and mechanistic contribution of EAAT2a in balancing brain excitability, and its direct link to epileptic seizures.
\end{abstract}

\section{INTRODUCTION}

Astroglia are the most numerous glial cells within the central nervous system (CNS). They do not only provide trophic support to neurons but also play an important role in synapse formation and neurotransmission ${ }^{1-4}$. By taking up neurotransmitters from the synaptic cleft, these glial cells are crucial for regulating synaptic transmission. The excitatory amino acid transporter 2 (EAAT2), expressed mainly on astroglia, plays a key role in synaptic regulation by removing the majority of extracellular glutamate, which is the main excitatory neurotransmitter in the CNS 5,6 . Impaired glutamate clearance by this transporter has been shown to lead to synaptic accumulation of the neurotransmitter, resulting in an overactive CNS and excitotoxicity ${ }^{7,8}$. This in turn may cause epilepsy, a group of brain disorders characterized by recurrent seizures ${ }^{9}$.

Previous studies have shown that astroglia-neuron interactions may play an essential role in seizure initia- tion and propagation ${ }^{10-12}$. On one hand, the functional coupling of astroglia through gap junctions is crucial to avert excessive neuronal activation, accomplished by rapid re-distribution of ions and neurotransmitters across the connected astroglial network ${ }^{12}$. On the other hand, this functional syncytium might also, under special circumstances, promote epileptogenesis ${ }^{13}$. The intercellular spread of calcium waves among astroglia can affect neuronal synchronization and therefore influence the propagation of seizure activity ${ }^{10}$. In addition, disruptions of the glutamate-glutamine cycle, in which EAAT2 is essential, are linked with temporal lobe epilepsy in human patients and rodents ${ }^{14}$. Accordingly, it is essential to understand how loss of EAAT2 affects neurons and astroglia.

In the present study, we generated a zebrafish (Danio rerio) mutant lacking EAAT2a, the zebrafish orthologue matching the mammalian EAAT2 in biophysical characteristics and mainly glial expression pattern ${ }^{15,16}$. Calcium imaging in the transparent zebrafish larvae allowed us to image whole-brain network activity in vivo ${ }^{17}$. We show that loss of EAAT2a transporter in larval zebrafish leads to increased brain excitability and recurrent spontaneous seizures, mimicking a human phenotype of patients with de novo mutations in EAAT2 ${ }^{18,19}$. These seizures are manifested in zebrafish larvae by epileptic locomotor bursts and periods of excessive brain activity, accompanied by increased extracellular glutamate concentrations. Counterintuitively, apart from these periods of hyperexcitation, neuronal and astroglial network activity of eaat $2 a^{-/}$mutants is reduced. This coincides with a decreased overall locomotion compared to their unaffected siblings, and mirrors slow background brain activity and reduced muscle tone present in human patients ${ }^{18,19}$. Altogether, our in vivo model of impaired EAAT2a function results in a depressed yet hyperexcitable brain state, and mimics a form of developmental and epileptic encephalopathy (DEE). 


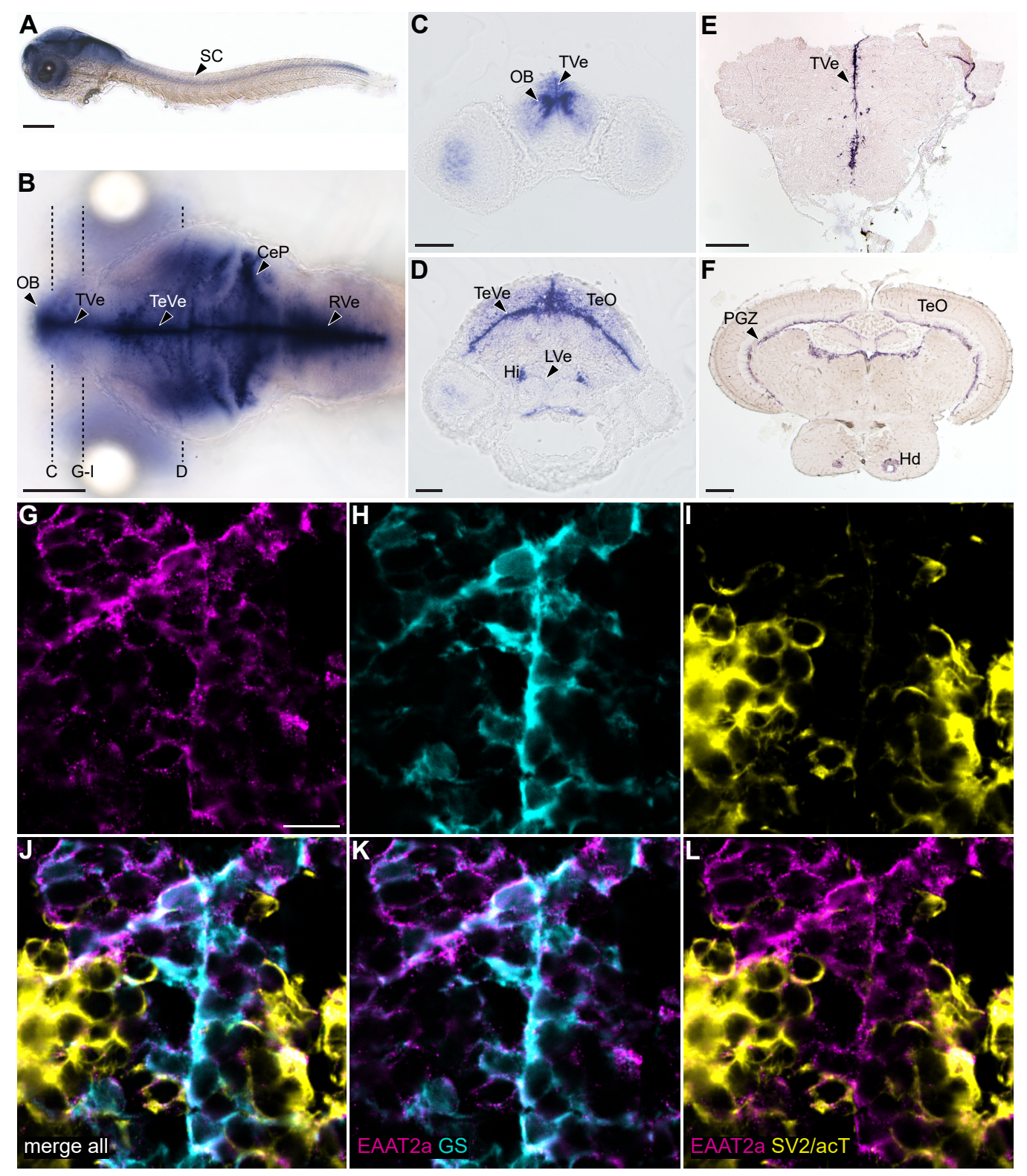

Figure 1: EAAT2a is predominantly expressed in astroglial cells. (A and B) mRNA of eaat $2 a$ is expressed along the periventricular zones (TVe, TeVe, RVe), resembling astroglial localization patterns in larval zebrafish as visible in lateral (A) and dorsal (B) view. (C and D) Cross sections of (B) indicated by dashed lines. ( $\mathbf{E}$ and $\mathbf{F}$ ) eaat 2 a mRNA expression along periventricular zones (TVe, PGZ) is maintained in adult zebrafish anterior forebrain (E) and midbrain (F). (G-I) Protein expression of EAAT2a (magenta), glutamine synthetase (GS, cyan) and synaptic vesicle 2/acetylated tubulin (SV2/acT, yellow) on cross sections of larval anterior forebrain indicated in (B). (J-L) Overlay of EAAT2a, GS and SV2/acT shows a greater co-localization of EAAT2a with astroglial $(\mathbf{K})$ than neuronal $(\mathbf{L})$ cells. $\mathrm{CeP}=$ cerebellar plate, $\mathrm{Hi}=$ intermediate hypothalamus, $\mathrm{Hd}=$ dorsal zone of periventricular hypothalamus, $\mathrm{LVe}=$ lateral ventricular recess of hypothalamus, $\mathrm{OB}=$ olfactory bulb nuclei, $\mathrm{PGZ}=$ periventricular gray zone of the optic tectum, $\mathrm{TVe}=$ periventricular zone of the telencephalon, $\mathrm{TeO}=$ optic tectum, $\mathrm{TeVe}=$ periventricular zone of the tectum, $\mathrm{RVe}=$ periventricular zone of the rhombencephalon. Scale bars are $200 \mu \mathrm{m}$ in $\mathbf{A}, \mathbf{B}, \mathbf{E} \& \mathbf{F} ; 50 \mu \mathrm{m}$ in $\mathbf{C}$ and $\mathbf{D} ; 10 \mu \mathrm{m}$ in $\mathbf{G}$.

\section{RESULTS}

\section{EAAT2a is predominantly expressed in astroglial} cells

To investigate the expression pattern of eaat $2 a$ transcripts in the larval zebrafish, we first performed in situ hybridization experiments. Our results showed that eaat $2 a$ transcripts are expressed in all parts of the CNS (Fig. 1A). Specifically, we observed eaat $2 a$ transcripts along the spinal cord (Fig. 1A), the periventricular zones of the forebrain, the midbrain, and the hindbrain (Fig. 1B-D). In adult fish, the high expression in the forebrain (Fig. 1E) and tectal periventricular regions was maintained (Fig. 1F).
These results suggest that spatial distribution of zebrafish eaat $2 a$ transcripts mainly overlaps with the location of astroglial cells, the functional homologues of mammalian astrocytes ${ }^{20,21}$. To further confirm the precise expression of eaat $2 a$, we performed triple staining using antibodies against astroglia and neuron specific proteins together with our custom made paralogue-specific antibody against EAAT2a ${ }^{16}$ (Fig. $1 G-L)$. We found substantial co-localization of EAAT2a staining with the astroglial glutamine synthetase (GS) along the ventricular zones (Fig. $1 \mathrm{~K})$, while neuronal cell labeling with SV2 (presynapse) and acetylated tubulin (axons) only 
showed weak EAAT2a signals (Fig. 1L). These results demonstrate that EAAT2a is mainly expressed in astroglial cells.

\section{Loss of EAAT2a leads to morphological defects and larval lethality}

To elucidate the function of EAAT2a in brain development and function, we generated CRISPR/ Cas9-mediated knockout (KO) mutants targeting exon 3 preceding the transmembrane domains involved in transport function ${ }^{22}$. The selected mutant allele harbors a -13 base pair deletion, leading to premature STOP codons within the third transmembrane domain (Supplementary Fig. 1). The predicted truncated protein fragment is devoid of functional transport domains, consequently no EAAT2a antibody signal was present in eaat $2 a^{-1-}$ mutants (Supplementary Fig. 1 and 2). We observed that eaat $2 a^{-\alpha}$ zebrafish larvae displayed an aberrant morphology. They failed to inflate their swim bladder, developed pericardial edemas and were smaller than their siblings (Fig. 2A). Body size measurements confirmed that at $3,4,5,6$, and 7 days post fertilization (dpf), eaat $2 a^{-/}$zebrafish were significantly smaller than control animals (Fig. 2B), with reduced brain size at $5 \mathrm{dpf}$ (Supplementary Fig.
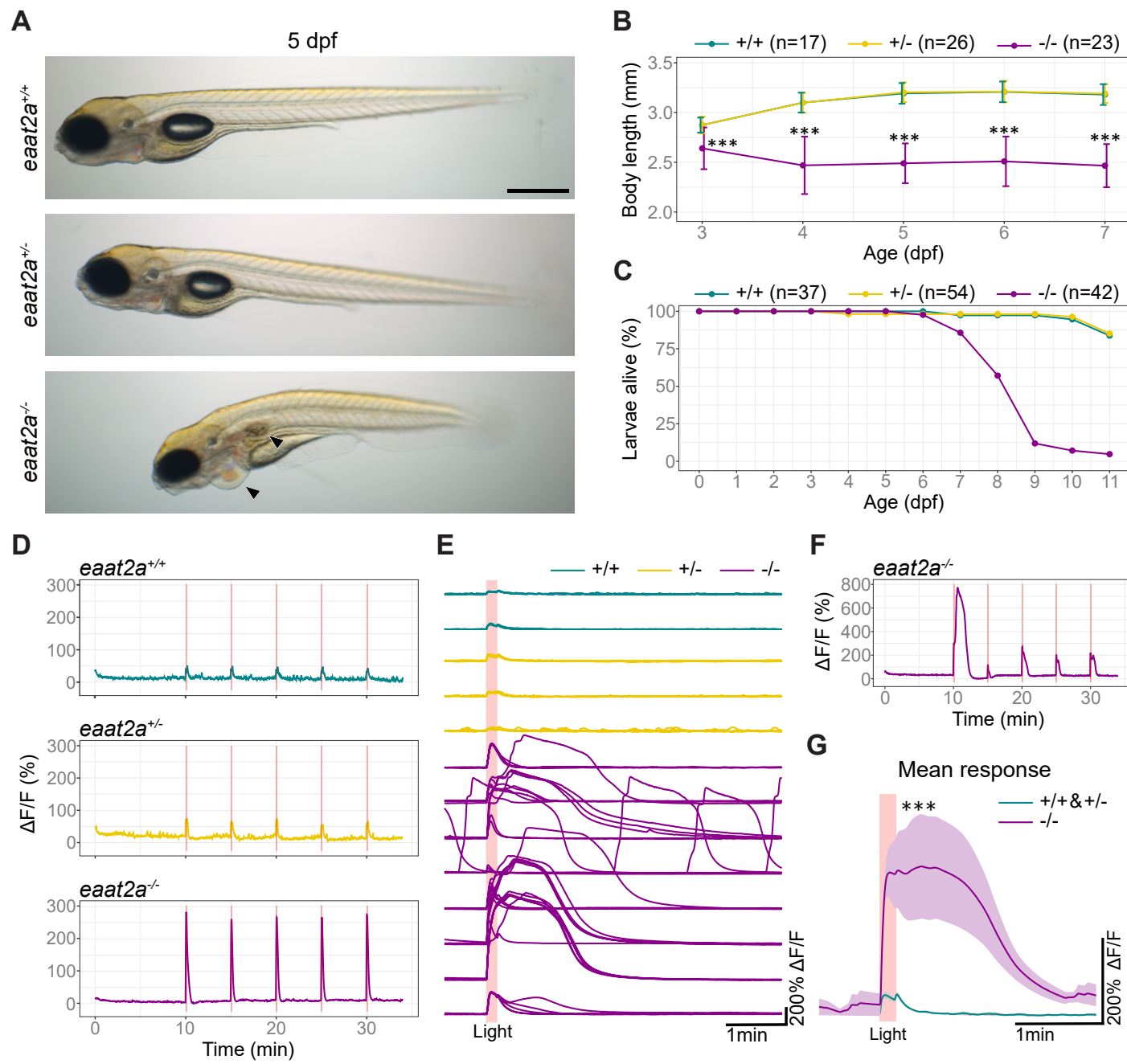

Figure 2: Knockout of eaat $2 a$ leads to hyperexcitability in response to light stimulation, morphological defects and larval lethality. (A) Lateral view of $5 \mathrm{dpf}$ eaat2a mutants. eaat $2 a^{-/}$larvae (bottom) are slightly curved, do not have an inflated swim bladder and develop pericardial edema (arrowheads). eaat $2 a^{+/+}$(top) and eaat $2 a^{+/}$(middle) larvae are indistinguishable. Scale bar
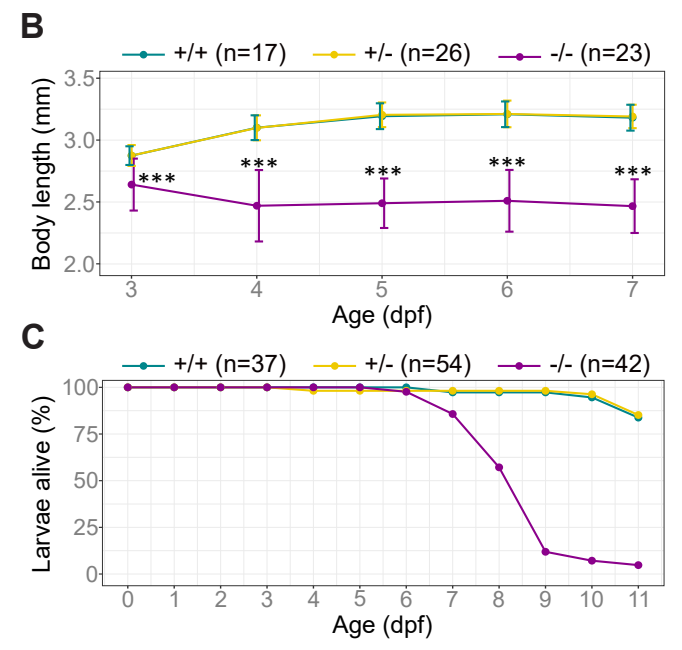

3). Furthermore, eaat $2 a^{-/}$mutants were not viable past larval stage and started to die from $6 \mathrm{dpf}$ on (Fig. $2 \mathrm{C})$. Only $10 \%$ of eaat $2 a^{-/-}$larvae were still alive at 9 $\mathrm{dpf}$, and remaining survivors were heavily impaired, displaying large edemas and reduced locomotion. In contrast, eaat $2 a^{+/}$heterozygotes were indistinguishable from eaat $2 a^{+/+}$siblings by visual

eaat $2 a^{-/}$mutant zebrafish exhibit hyperexcitability in response to light stimulation

EAAT2 is an essential part of the glutamate clearance mechanism in the brain ${ }^{5,16}$. Hence, the absence of the glutamate transporter may lead to changes in neuronal excitability in eaat $2 a^{-/}$mutant zebrafish. To test this hypothesis, we compared brain-wide neuronal responses to transient light flashes in eaat $2 a^{-/}$zebrafish and control siblings expressing the transgenic calcium indicator GCaMP6s under the neuronal elavl3 promoter ${ }^{10}$. We observed that eaat $2 a^{-}$ mutant larvae displayed highly amplified light responses compared to their eaat $2 a^{+/-}$and eaat $2 a^{+/+}$ siblings (Fig. 2D and E), with varying response mplitudes across and within eaat $2 a^{-/}$larvae (Fig. 2F). Strikingly, average neuronal responses in eaat $2 a^{-/}$larvae were not only excessively enlarged, 
is $500 \mu \mathrm{m}$. (B) Spinal cord length analysis of eaat 2 a mutants at consecutive days reveals a smaller body size in eaat $2 a^{-/-}$(magenta) compared to their eaat $2 a^{+/}$(yellow) and eaat $2 a^{+/+}$(cyan) siblings. (C) Survival curve of eaat2a mutants. (D) Whole-brain neuronal activity (elavl3:GCaMP6s signal) of three representative eaat 2 a mutant larvae (eaat $2 a^{+/+}$in cyan, eaat $2 a^{+/-}$in yellow and eaat $2 a^{-/}$ in magenta) exposed to five 10-second light stimuli with 5-minute interstimulus interval. (E) Changes in fluorescence over time $(\Delta \mathrm{F} / \mathrm{F})$ per larvae aligned at the onset of light stimuli. $(\mathrm{F}) \Delta \mathrm{F} / \mathrm{F}$ trace of an example eaat $2 a^{-/}$larvae with diverse responses to lightstimuli. (G) Mean responses to light stimuli in $5 \mathrm{dpf}$ eaat $2 a^{+/+}$and eaat $2 a^{+/}$(cyan), and eaat $2 a^{-/}$(magenta) zebrafish larvae. Shaded area represents SEM. Significance level: ${ }^{* * *} p=<0.001$, Kruskal Wallis test with Wilcoxon rank-sum post hoc test (B) or Wilcoxon rank-sum test $(\mathbf{G})$.

but also very long lasting (Fig. 2G). Occasionally, we also observed spontaneous neuronal activity bursts of similar magnitude (Fig. 2E), potentially resembling spontaneous epileptic seizures ${ }^{9,23-25}$. Taken together, our results show that eaat $2 a^{-/}$zebrafish brains exhibit increased excitability in responses to light stimulation and are possibly prone to epileptic seizures.

\section{eaat $2 a^{-1-}$ mutant zebrafish show spontaneous seizures coinciding with a surge of extracellular glutamate}

Our neuronal activity recordings suggested that eaat $2 a^{-\alpha}$ mutant zebrafish not only exhibit strong light-induced responses but also display occasional neuronal activity bursts resembling epileptic seizures. To further characterize this spontaneous seizure-like phenotype in eaat $2 a^{-/}$mutants, we examined swimming behavior in $5 \mathrm{dpf}$ larvae by using automated behavioral tracking. We observed that eaat $2 a^{+/+}$and eaat $2 a^{+/}$zebrafish swim with periods of stop, slow and fast swims as described previously in healthy zebrafish 26,27. In contrast, eaat $2 a^{-1-}$ mutant animals displayed aberrant locomotor patterns (Fig. 3A-F). eaat $2 a^{-/}$larvae swam substantially less than their eaat $2 a^{+/}$and eaat $2 a^{+/+}$ siblings, mainly lying motionless on the bottom of the dish (Fig 3A-C). Strikingly, when eaat $2 a^{-/}$larvae swam, they showed convulsive twitching and swim bursts occasionally followed by swirling around their body axis and finally immobilized sinking to the bottom of the dish (Supplementary Movie 1). Compared to their siblings, eaat $2 a^{-/}$zebrafish showed more swim bursts of either long distance (Fig. 3D and E) or high velocity during a prolonged period (Fig. 3F, Supplementary Fig. 4). This swirling and bursting behavior highly resembles established models of epilepsy and is interpreted as seizure-like behavior in zebrafish ${ }^{24,28}$. All these findings support the idea that eaat $2 a^{-1}$ larvae exhibit spontaneous seizures.

To verify the presence of seizure-activity in eaat $2 a^{-/-}$ mutant brains, we measured local field potentials (LFPs) by inserting a micro-electrode in the anterior forebrain (telencephalon) ${ }^{10}$ of $5 \mathrm{dpf}$ animals. As expected, the measured electrical activity in eaat $2 a^{-/}$ larvae revealed spontaneous episodes of high voltage LFP deflections (Fig. 3G), resembling seizure-like LFP activity in other zebrafish models ${ }^{10,29,30}$. These spontaneous seizures were not detected in eaat $2 a^{+-}$or eaat $2 a^{+/+}$siblings (Fig. $3 \mathrm{G}$ and $\mathrm{H}$ ). We hypothesized that spontaneous seizures in eaat $2 a^{--}$mutant larvae are associated with an excess of extracellular glutamate that cannot be removed due to impaired glutamate clearance ${ }^{31}$. Hence, we expected to see large glutamate surges during spontaneous seizures. To test this hypothesis, we combined LFP measurements with simultaneous fluorescence recordings of extracellular glutamate near astroglial terminals using $T g$ (gfap:iGluSnFR) animals ${ }^{32}$. In eaat $2 a^{-/}$larvae, we observed massive increases of iGluSnFR signal reflecting glutamate levels, coinciding with spontaneous LFP-seizures (Fig. 3I). No such large glutamate surges were observed in eaat $2 a^{+/}$and eaat $2 a^{+/+}$control siblings. Taken together, our results show that loss of EAAT2a leads to spontaneous seizures that coincide with a massive surge in extracellular glutamate levels.

\section{Neuronal hyperactivity during seizures contrasts with basal hypoactivity in eaat $2 a^{-/}$larvae}

To characterize EAAT2a-related seizures further, we investigated the neuronal activity in $5 \mathrm{dpf}$ Tg(elavl3:GCaMP5G) zebrafish. Our recordings revealed recurrent periods of excessive neuronal activity spreading across the entire brain of eaat $2 a^{-/}$ mutants (Fig. 4A, Supplementary Movie 2 and 3). During these spontaneous seizures, neuronal calcium signals across the brain reached levels greater than $100 \%$ of relative change in fluorescence $(\Delta \mathrm{F} / \mathrm{F})$ (Fig. 4B bottom). These globally high levels of neuronal seizure-activity were maintained for more than a minute before decreasing to a short hypoactive period, and finally returning to inter-ictal (between seizures) levels (Fig. 4B bottom and 4C). These results confirm that eaat $2 a^{-/}$mutants exhibit spontaneous global seizures that are not present in eaat $2 a^{+/-}$and eaat $2 a^{+/+}$control siblings (Fig. 4D). In addition to these global seizures, we found that some larvae additionally or exclusively showed localized seizures not reaching the anterior forebrain and lasting for less than a minute (Supplementary Fig. 5). Next, we asked how different brain regions are recruited during seizure propagation in eaat $2 a^{-/}$ mutants. As represented in Figure 4E, excessive increase in intracellular calcium levels was initiated in the midbrain (8 of 12 fish) or hindbrain (4 of 12 fish). To quantify this further, we compared half 


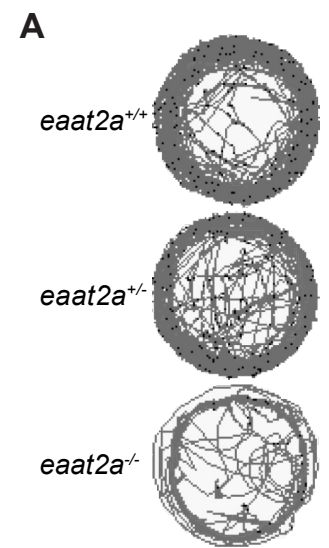

C

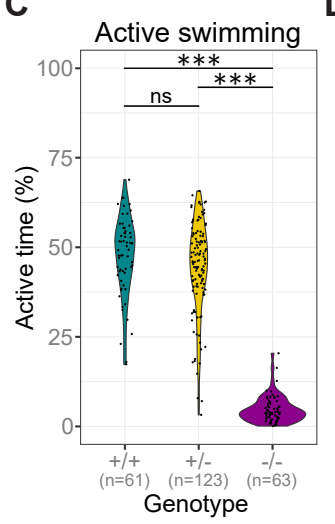

G

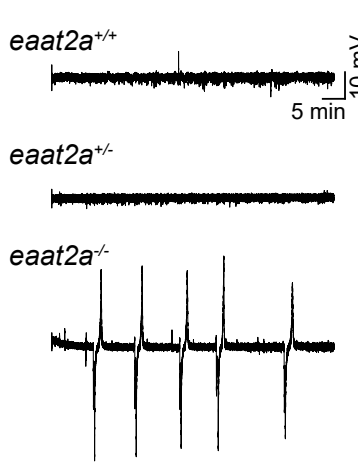

B

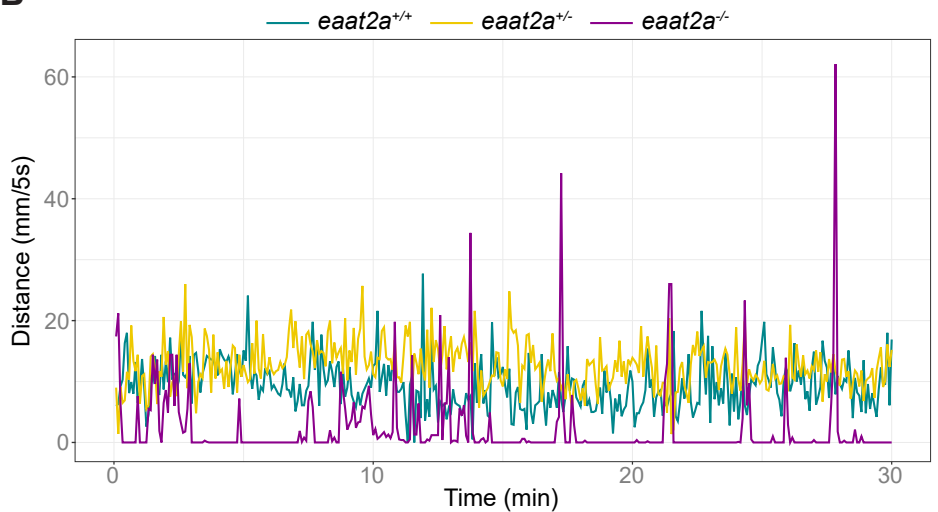

$F$

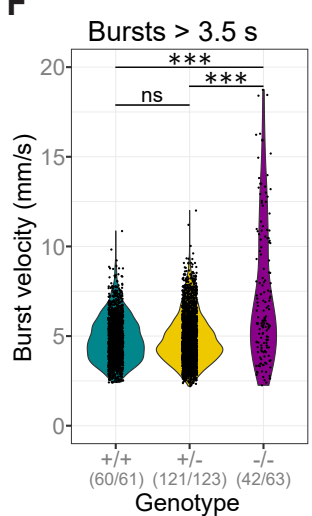

H

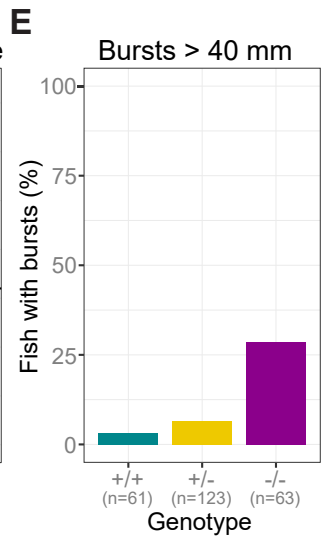

I
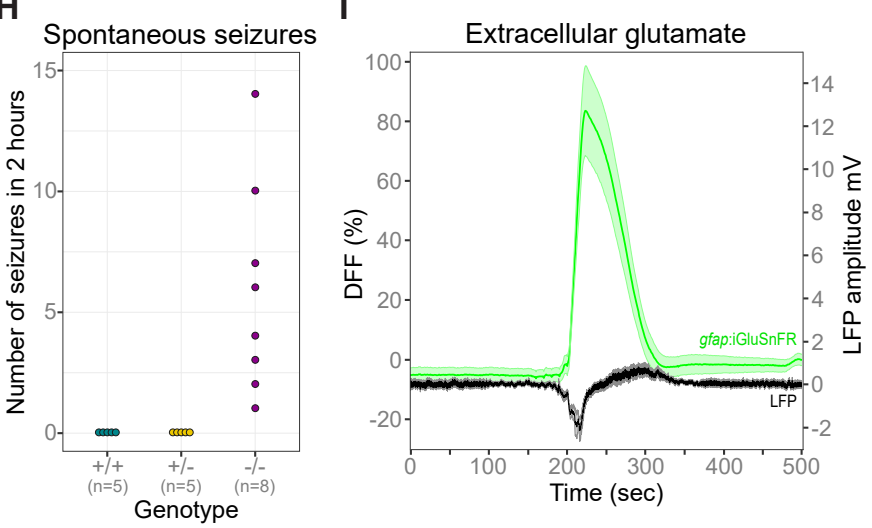

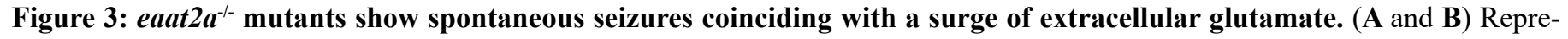
sentative traces for swim location (A) and distances (B) over 30 minutes in $5 \mathrm{dpf}$ eaat $2 a^{-/}$(magenta), eaat $2 a^{+/}$(yellow) and eaat $2 a^{+/+}$(cyan) larvae. (C) Ratio of time period with active swimming, per genotype. (D) Distance moved per five-second integral during 30-minute recordings, per genotype. Black dotted line represents threshold $>40 \mathrm{~mm} / 5 \mathrm{~s}$. (E) Proportion of fish showing one or more bursts bigger than $40 \mathrm{~mm}$ during five-second integrals. (F) Velocity of all bursts lasting longer than 3.5 seconds. (G) Representative telencephalic local field potential (LFP) recordings. (H)Number of spontaneous global seizures detected during twohour LFP recordings. (I) Average change in iGluSnFR fluorescence $(\Delta \mathrm{F} / \mathrm{F}$, green) and simultaneous local field potential (LFP, black) recordings during epileptic activity in $5 \mathrm{dpf}$ eaat $2 \mathrm{a}^{-/}$mutants. $\mathrm{n}=8 \mathrm{fish}$. Signals are aligned at the onset of global seizures. Significance levels: ${ }^{* * *} p=<0.001$, ns $=$ not significant $(p>0.05)$, Kruskal-Wallis rank-sum test with Wilcoxon rank-sum posthoc test (C) or two-sample Kolmogorov-Smirnov test (F).

maxima of neuronal calcium signals $(\Delta \mathrm{F} / \mathrm{F})$ between anterior forebrain, midbrain and hindbrain during global seizures lasting for more than one minute. We observed that neurons of the anterior forebrain were recruited only seconds after seizure initiation (mean $13 \mathrm{sec}, \mathrm{SD} \pm 5.67 \mathrm{sec}$ ), regardless of the seizure origin (Fig. 4F). Beyond these spontaneous seizures, we also observed that the large amplitude light responses shown earlier in Fig. 2E were very similar to spontaneous global (Fig. 4G, curly brackets) and localized (Fig. 4G, square brackets) seizures with comparable amplitudes (Fig. 4H). Since epileptic seizures that are objectively and consistently evoked by a specific external stimulus are referred to as reflex seizures, we termed these excessive responses to light in eaat $2 a^{-/}$mutants as light-induced reflex seizures ${ }^{33}$. Intriguingly, during inter-ictal periods, eaat $2 a^{-/-}$ mutants exhibited reduced basal neuronal activity compared to eaat $2 a^{+/-}$and eaat $2 a^{+/+}$siblings (Fig. 4I). We observed smaller fluctuations (standard deviation) of neuronal calcium signals in eaat $2 a^{-/}$ larvae compared to their siblings (Fig. 4J). This reduced basal activity might explain hypoactive locomotor behaviors of eaat $2 a^{-/-}$mutants (Fig. 3C). 
Taken together, our results indicate that loss of EAAT2a not only leads to spontaneous seizures, but also reduces basal neuronal activity during inter-ictal periods. Moreover, in all our experiments, eaat $2 a^{+/}$ mutants were indistinguishable from their eaat $2 a^{+/+}$ siblings, suggesting that eaat $2 a^{+/}$mutation does not lead to haploinsufficiency or any seizure phenotype.

\section{Astroglial network in eaat $2 a^{-/-}$mutants is silent yet hyperexcitable}

Given the predominant astroglial expression of EAAT2a, one potential cause for the reduced neuronal basal activity in eaat $2 a^{--}$mutants may be the impaired astroglial glutamate recycling, which reduces available glutamate. To investigate whether astroglial function is impaired in eaat $2 a^{-/}$mutants, we recorded glial calcium signals in $5 \mathrm{dpf}$ larvae

A

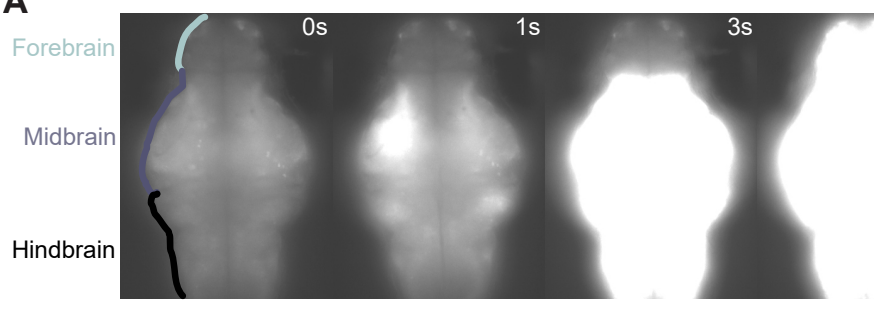

B

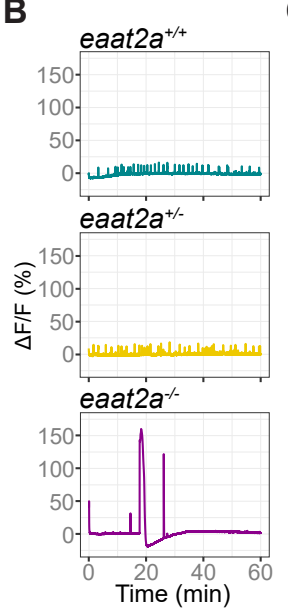

G

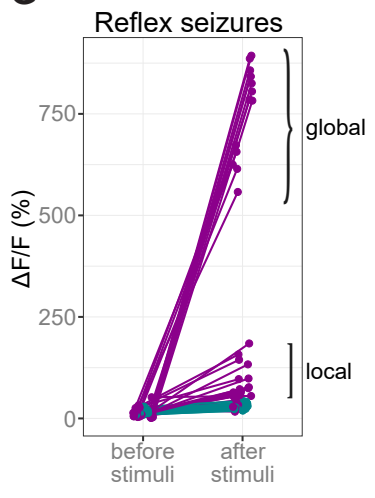

H

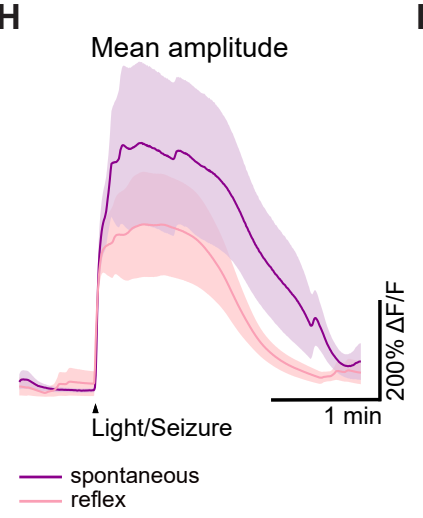

C

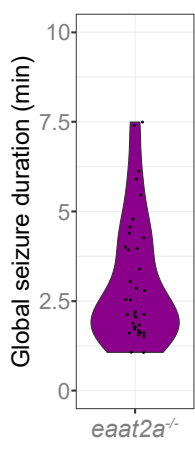

D

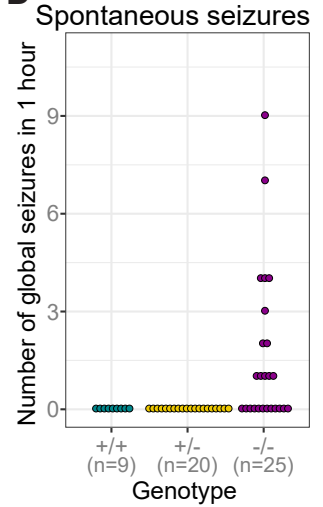

E

I

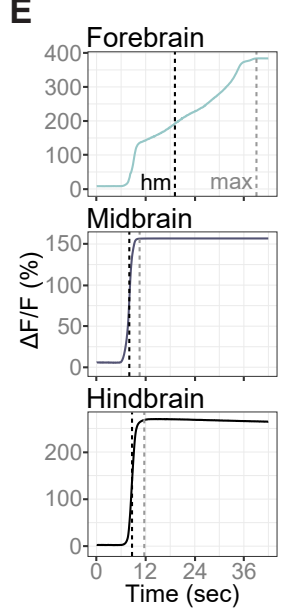

F
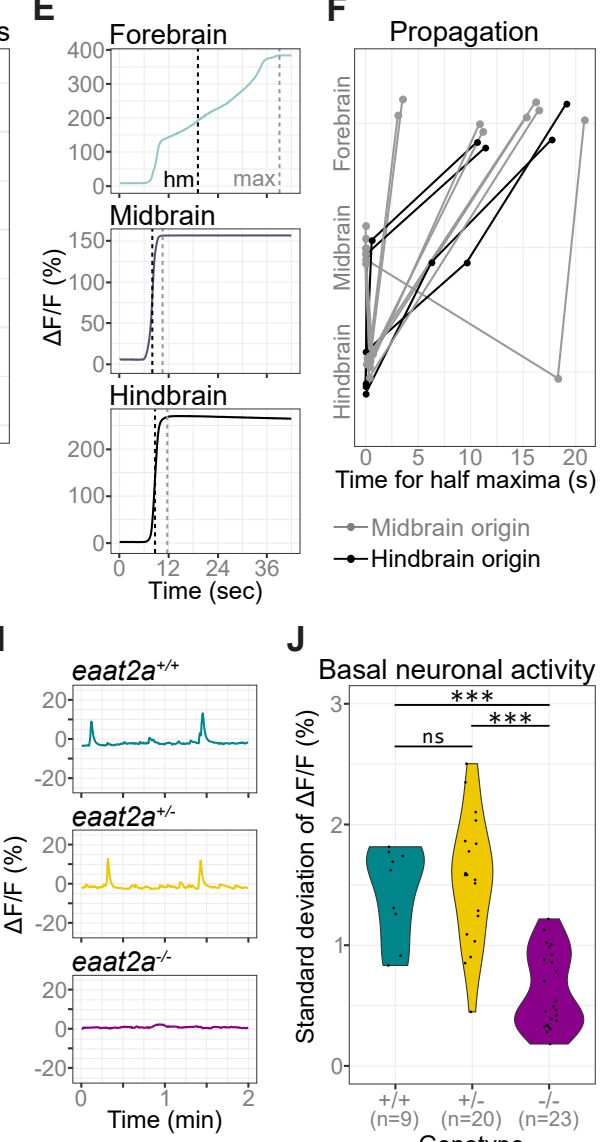

J

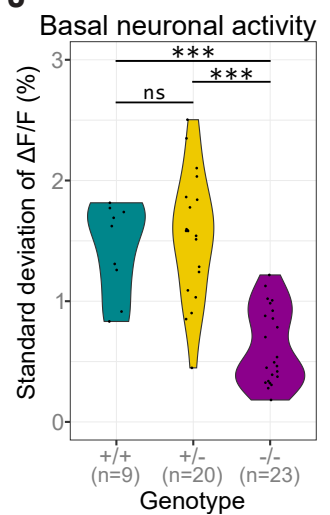

Figure 4: Neuronal hyperactivity during seizures contrasts with basal hypoactivity in eaat $2 a^{-/}$larvae. (A) Time lapse of neuronal calcium signals during a seizure in eaat $2 a^{-/}$larva in $\operatorname{Tg}($ elavl3:GCaMP5G) background (dorsal view). Scale bar is $100 \mu \mathrm{m}$. (B) Representative calcium signals (elavl3:GCaMP5G) recorded across the brain of eaat $2 a^{+/+}$(cyan, top), eaat2a ${ }^{+/}$(yellow, middle) and eaat $2 a^{-/}$(magenta, bottom) larvae. (C) Duration of spontaneous global seizures present in eaat $2 a^{-/}$mutants (median 2 min 7 $\sec (\operatorname{std} \pm 52 \mathrm{sec}))$. (D) Number of spontaneous global seizures recorded by calcium imaging during 60 minutes. (E) Neuronal activity (elavl3: GCaMP5G) of the three main brain regions of a representative global seizure. The half maxima (hm) represents the time point of $\max (\Delta \mathrm{F} / \mathrm{F}) / 2$. (F) Relative time for $\Delta \mathrm{F} / \mathrm{F}$ half maxima represents propagation of global seizures across brain parts over time. Colors indicate region of seizure origin: light grey for midbrain, black for hindbrain. $\mathrm{n}=12$ eaat $2 a^{-/}$larvae. (G) Calcium signals (elavl3:GCaMP6s) one minute before and immediately after light stimuli. Brackets indicate global (curly brackets) and local (square brackets) reflex seizures. (H) Averaged calcium signals for spontaneous (magenta) and reflex (light pink) seizures during light-stimuli recordings. Shaded area represents SEM. (I) Magnification of (B) shows calcium signals (elavl3:GCaMP5G) during two-minute basal activity period used for standard deviation calculations in $(\mathbf{J})$. (J) Neuronal basal activity calculated by the standard deviation of $\Delta \mathrm{F} / \mathrm{F}$ over two minutes in eaat $2 a^{-/}$mutants (mean $\left.0.63, \mathrm{n}=23\right)$ compared to their eaat $2 a^{+/}(\mathrm{mean} 1.54, \mathrm{n}=20, p$ $=2.7 \mathrm{e}-07)$ and eaat $2 a^{+/+}$(mean 1.44, $\left.\mathrm{n}=9, p=1 \mathrm{e}-04\right)$ siblings. Significance levels: $* * * p=<0.001$, ns $=$ not significant $(p=>0.05)$, Kruskal Wallis test with Wilcoxon rank-sum posthoc test (I). 
expressing GCaMP6s under the glial promoter gfap (Tg(gfap:Gal4)nw7; $\operatorname{Tg}\left(\right.$ UAS:GCaMP6s)) ${ }^{10}$. $\quad$ To exclusively analyze astroglial activity, we focused on the region along the ventricular zones in the forebrain and midbrain (Fig. 5A) ${ }^{10}$. In line with our previous results, we observed spontaneous events of excessive astroglial activity $(\Delta \mathrm{F} / \mathrm{F}$ greater than $100 \%)$ spreading across the entire brain of eaat $2 a^{-/}$mutants (Fig. 5B), often finally recruiting the anterior forebrain (Fig. 5C, Supplementary Movie 4 and 5). These events likely represent astroglial activity during seizures ${ }^{10}$. Such spontaneous global events were prominent at least once per hour in eaat $2 a^{-/-}$ mutants and absent in eaat $2 a^{+/}$and eaat $2 a^{+/+}$control siblings (Fig. 5D). To further characterize astroglial activity, we quantified the ratio of active glial cells and the amplitudes per calcium burst in eaat $2 a^{-1-}$ mutant larvae during inter-ictal, pre-ictal (preceding seizure onset) and ictal periods. We observed a drastic increase in the ratio of active astroglia and amplitude of calcium bursts at the transition from pre-ictal to ictal period, while inter-ictal and pre-ictal periods appeared to be similar (Fig. 5E and F). Next, we tested whether these large bursts of astroglial calcium signals are also present in light-evoked seizures. Our results revealed that the ratio and the amplitude of astroglial calcium signals were significantly larger in eaat $2 a^{-/-}$mutants, when compared to control siblings (Fig. 5G and H). Furthermore, some of these lightevoked seizures propagated globally, recruiting the entire brain including the anterior forebrain (Fig. 5H, arrowheads). During inter-ictal periods, a smaller ratio of astroglial cells were active (Fig. 5I), and these active cells had reduced overall activity (Fig. 5J) in eaat $2 a^{-1}$ mutants compared to their siblings'. However, we did not observe a significant difference in the amplitude or the duration of individual astroglial calcium bursts between eaat $2 a^{-1-}$ mutants and control siblings during inter-ictal/basal periods (Fig. 5K and L). Taken together, our results revealed that astroglial cells in eaat $2 a^{-/}$mutants are less active during inter-ictal states, and generate excessive calcium bursts only during seizures.

The depressed yet hyperexcitable brain state of eaat $2 a^{-/}$mutants implies pathological changes in the brain. To test the impact of eaat $2 a$ loss on the gene expression of key regulators of neuronal and astroglial activity, we analyzed whole-brain transcript levels using quantitative reverse transcription PCR (RT-qPCR). Firstly, we tested for transcriptional adaptation of other eaat glutamate transporters. Expression levels of eaat $2 b$, the eaat $2 a$ paralogue, did not differ in eaat $2 a^{--}$animals (Fig. 5M). In contrast, another glutamate transporter eaat $1 b$ was upregulated in eaat $2 a^{-/}$mutants compared to eaat $2 a^{+/+}$siblings (Fig. 5M), suggesting a compensatory effect to improve buffering of excess synaptic glutamate in eaat $2 a^{-/}$mutants (Fig. 3I).
Secondly, we assessed whether expressions of genes associated with inhibitory neurotransmission of $\gamma$ aminobutyric acid (GABA) are altered. We found that neither the expression of the $\mathrm{GABA}_{\mathrm{A}}$ receptor subunit gabral nor the presynaptic enzyme gadlb differed considerably between eaat $2 a^{-/}$and eaat $2 a^{+/+}$ brains (Fig. 5M). Finally, we investigated whether metabotropic glutamate receptors (mGluRs) on astroglia are affected. These receptors can influence intracellular calcium transients in astroglia following synaptic glutamate release ${ }^{3}$. In eaat $2 a^{-/-}$larval brains, we observed no difference in expression levels of glial expressed mglur 3 or brain abundant ${ }^{34}$ mglur $5 b$. This suggests that reduced inter-ictal activity in eaat $2 a^{-\alpha}$ mutant astroglia is not due to impaired mGluR-induced calcium signaling. Taken together, the altered gene expression levels of eaat $1 b$ in eaat $2 a^{-/}$mutants suggest a compensatory attempt to adjust increased brain excitability by buffering glutamate.

\section{DISCUSSION}

In sum, we show that glutamate transporter EAAT2a is required to balance brain excitability by regulating extracellular glutamate levels. Our results indicate that impaired EAAT2a function results in epileptic seizures but also reduced basal brain activity (Fig. 6).

Epileptic seizures are due to an imbalance between excitatory and inhibitory synaptic transmission. In this study, we show that a genetic perturbation of the excitatory glutamate system leads to profound functional alterations in neuronal and astroglial networks, leading to spontaneous and light-induced seizures. Our results are in line with the earlier observations associating EAAT2 malfunctions to seizures in mice and humans ${ }^{18,19,35-39}$. In addition, our novel eaat $2 a^{-/-}$ mutant zebrafish epilepsy model has several important characteristics. Firstly, homozygous mutations of the astroglial eaat $2 a$ in zebrafish resemble a form of DEE present in human epilepsy patients with de novo mutations in the orthologous gene (EAAT2 = SLC1A2) ${ }^{18,19,37}$. DEE is clinically defined as a condition where developmental defects due to a mutation as well as epileptic activity itself contribute to the impairments ${ }^{40}$. The presence of both recurrent spontaneous seizures and severe developmental abnormalities in eaat $2 a^{-/}$mutants mirror the human phenotype 18,19. Secondly, spontaneous recurrent seizures in our eaat $2 a^{-/}$mutants enable the investigation of epileptogenesis throughout development. Although eaat $2 a^{-/}$ mutants' premature death restricts analysis to larval stages, future work on an inducible gene knockout would allow studies at more advanced developmental stages ${ }^{41}$. Finally, knockout of EAAT2a directly targets mainly astroglial networks, which are of great importance during seizure initiation and propagation 10-12. Therefore, our model will enable further investigations into the role of glia-neuron interactions in 
A

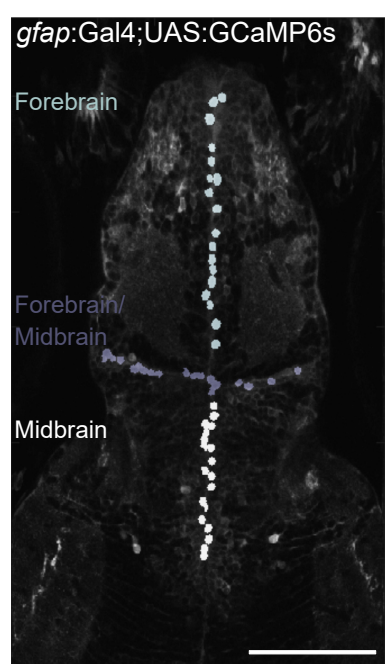

B
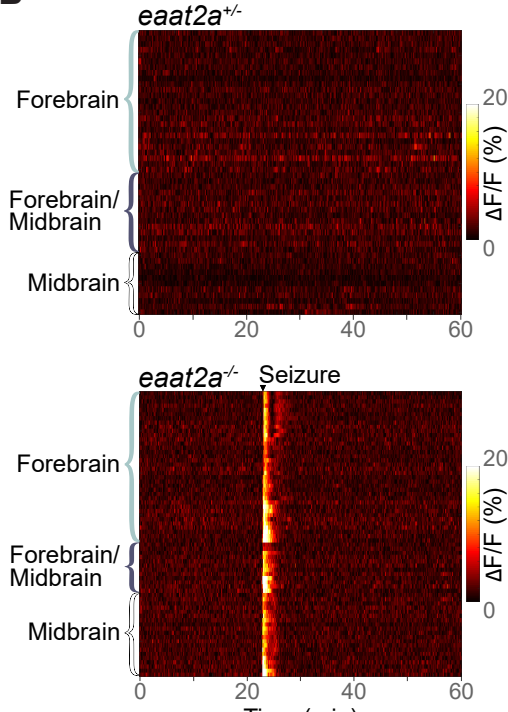

C
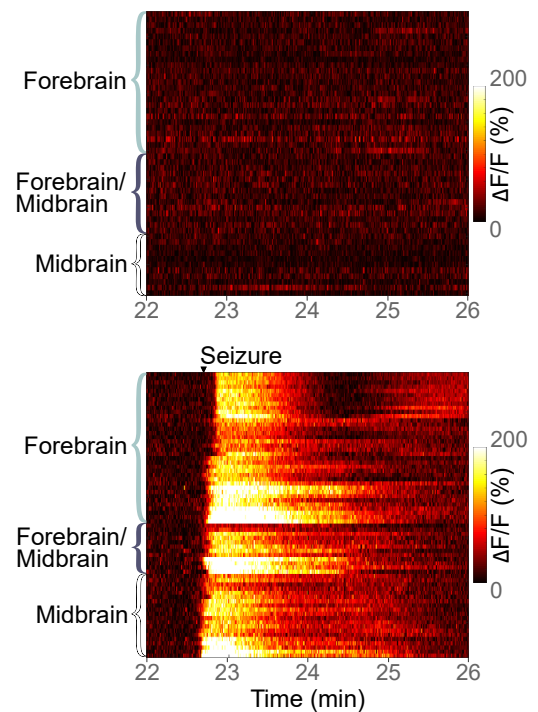

$\mathrm{H}$

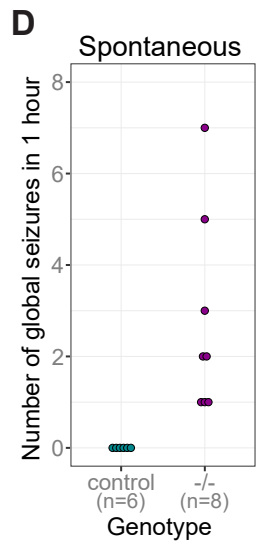

E

$\mathbf{F}$

G
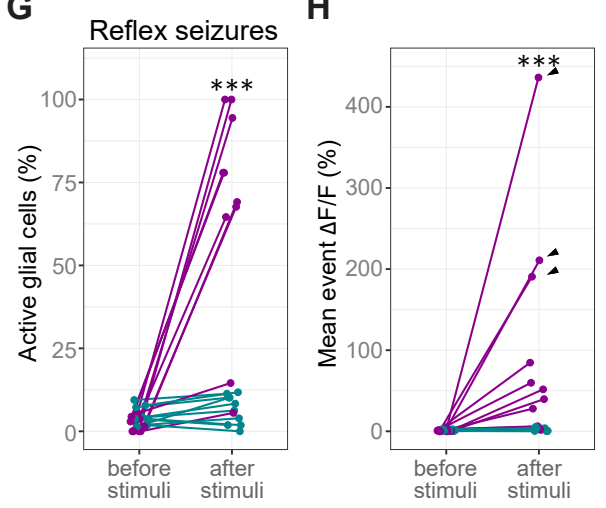

M
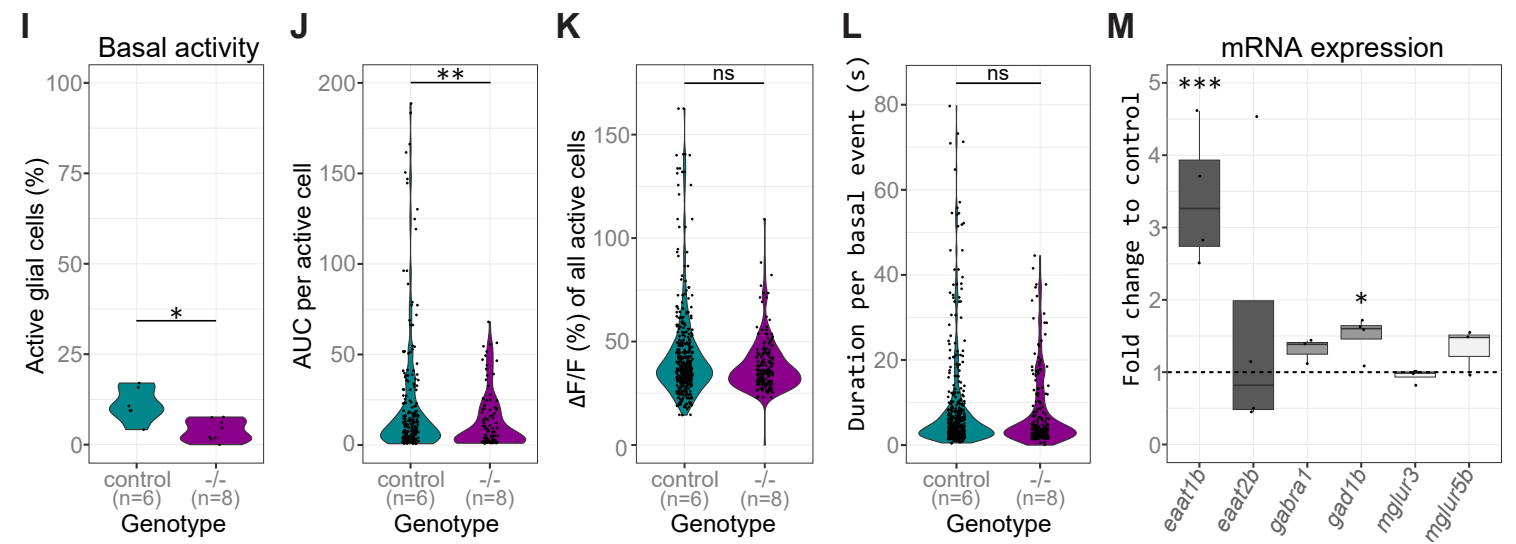

igure 5: Astroglial network in eaat2a/^ mutants is silent yet hyperexcitable. (A) Two-photon microscopy image of the forebrain and midbrain in a $5 \mathrm{dpf} T g$ (gfap:Gal4;UAS:GCaMP6s) zebrafish larva expressing GCaMP6s in GFAP positive glial cells. Individual astroglia along the ventricular regions are color-coded according to three different areas: forebrain (light grey), forebrain/midbrain boundary region (dark grey), and midbrain (white). Scale bar is $100 \mu \mathrm{m}$. (B) Calcium signals $(\Delta \mathrm{F} / \mathrm{F})$ of individual glial cells over time in representative eaat $2 a^{+-}$(top) and eaat $2 a^{--}$(bottom) larvae. Warm color indicates high activity as seen during the seizure in the eaat $2 a^{-\frown}$ larva. (C) Four-minute periods of calcium signals in (B). (D) Number of global seizures detected in eaat $2 a^{-\prec}$ mutants (magenta) compared to their eaat $2 a^{+/}$and eaat $2 a^{+/+}$siblings (cyan). (E and $\left.\mathbf{F}\right)$ Percentage of active glial cells (E) and average event amplitudes of active cells $(\mathbf{F})$ of individual eaat $2 a^{-/}$mutants during two-minute basal, preictal and ictal (spontaneous seizures) periods (E: $\left.p_{b-p}=1, p_{b-i}=0.0078, p_{p-i}=0.0078 ; \mathbf{f}: p_{b-p}=0.95, p_{b-i}=0.0078, p_{p-i}=0.0078\right)$. Cyan dots show average basal values of eaat $2 a^{+/+}$and eaat $2 a^{+/}$siblings. ( $\mathbf{G}$ and $\left.\mathbf{H}\right)$ Evaluation of one-minute periods immediately before and after 10-second light stimuli in eaat $2 a^{+/} /$eaat $2 a^{+/}$(cyan) and eaat $2 a^{-/}$(magenta) animals of the proportion of active cells $(\mathbf{G}, p=8.7 \mathrm{e}-4)$ and averaged amplitude over all cells $(\mathbf{H}, p=6.7 \mathrm{e}-4)$. Arrowheads indicate global seizures. $\mathrm{n}=5$ fish per group, 2 stimuli per fish. (I-L) Analysis during inter-ictal basal periods of percentage active astroglia per fish $(\mathbf{I}, p=0.011)$, total activity of active cells $(\mathbf{J}$, area under the curve $=$ AUC, $p=0.0011)$, amplitudes $(\mathbf{K}, p=0.052)$ and durations $(\mathbf{L}, p=0.37)$ of individual calcium bursts in active glial cells are plotted in violin plots with individual data points (black). $\mathrm{n}\left(\right.$ eaat $2 a^{++} /$eaat $\left.2 a^{+/}\right)=6, \mathrm{n}\left(\right.$ eaat $\left.2 a^{-/}\right)=8$. (M) $\mathrm{mRNA}$ transcript levels of eaat $1 b$ $(p=0.00075)$, eaat $2 b(p=0.94)$, gabral $(p=0.40)$, gadlb $(p=0.024)$, mglur3 $(p=0.86)$ and $m g l u r 5 b(p=0.40)$ in 5 dpf eaat $2 a^{-/}$ relative to eaat $2 a^{+/+}$siblings. Transcripts were measured by RT-qPCR and normalized to $g 6 p d$ and $b 2 m$. Significance levels: $* * * p$ $=<0.001,{ }^{* *} p=<0.01,{ }^{*} p=<0.05$, ns $=$ not significant $(p=>0.05)$, Wilcoxon signed rank test $(\mathbf{E}, \mathbf{F})$, Welch two sample unpaired t-test $(\mathbf{I}, \mathbf{M})$ or Wilcoxon rank-sum test $(\mathbf{G}, \mathbf{H}, \mathbf{J}-\mathbf{L})$. 
epilepsy. All these points give a novel vantage point for modelling and understanding potential mechanisms underlying human genetic epilepsies.

Recent studies show that astroglia and their interactions with neurons play an essential role in epilepsy ${ }^{10-12,42}$. The role of astroglial gap junctions in redistributing ions and neurotransmitters in epilepsy models are well investigated ${ }^{12,43}$. However, the function of astroglial glutamate transporters in epilepsy are less understood. Our results provide direct evidence for the importance of astroglial glutamate transporters for balancing brain excitability. On the one hand, in the absence of glutamate transporter EAAT2a, which is predominantly expressed on astroglia, we observed spontaneous seizures recruiting both neuronal and astroglial networks. In addition, light-induced reflex seizures are apparent in both cellular networks. Such evidence of hyperexcitability is likely due to impaired glutamate clearance in eaat $2 a^{-/}$mutants, leading to a massive transient surge of extracellular glutamate ${ }^{31}$, as measured by iGluSnFR imaging. The observed upregulation of glutamate transporter eaat $1 b$ transcripts further supports this hypothesis. On the other hand, we observed that both neuronal and astroglial networks are hypoactive during interictal periods. We argue that this hypoactive state might reflect the reduced availability of glutamate
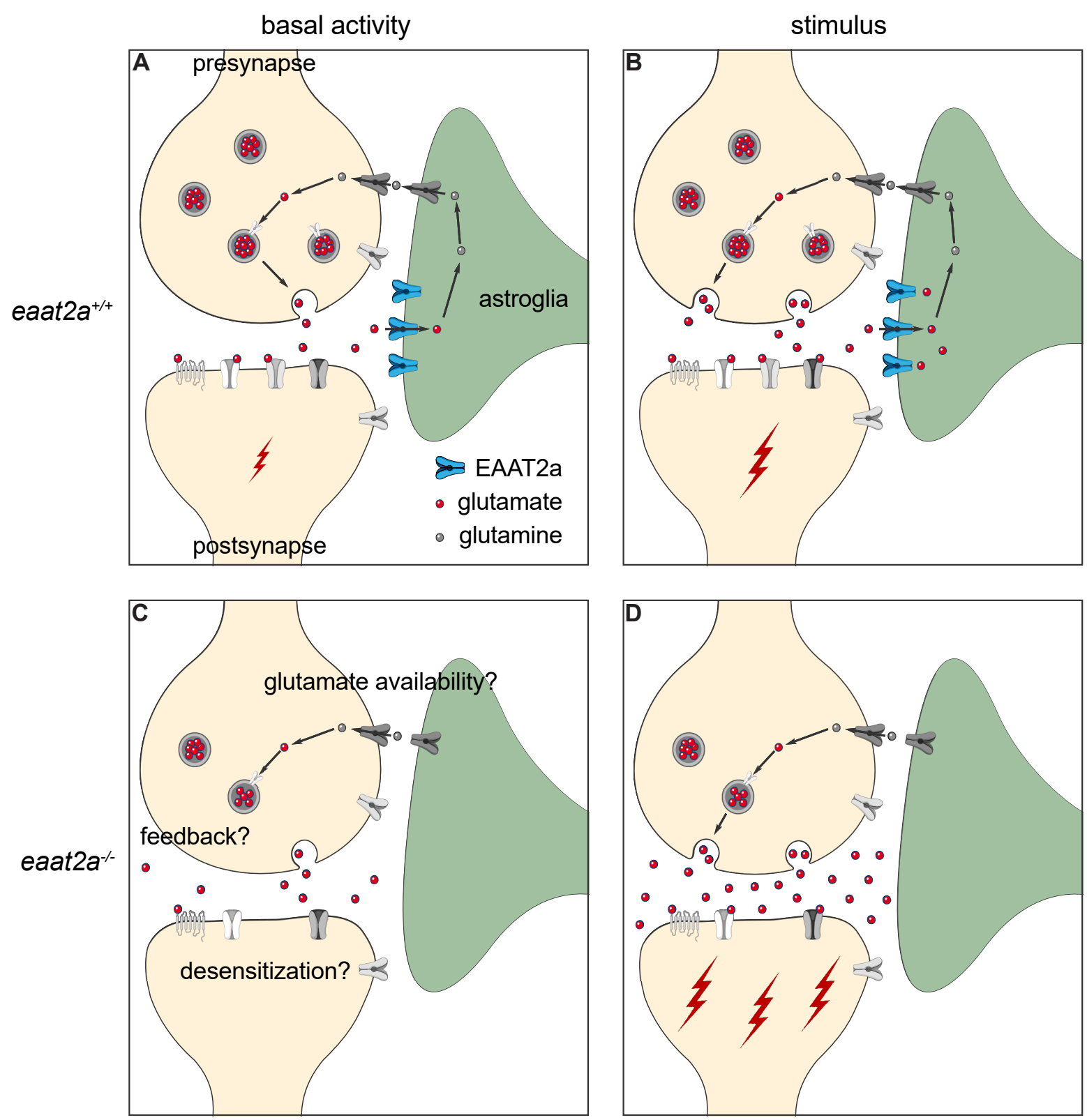

Figure 6: Working model of coexisting hyper- and hypoactivity in eaat2a ${ }^{-/}$mutants. (A and B) Glutamate transporter EAAT2a is important for both clearance of glutamate at the synaptic cleft and recycling of glutamate to the presynapse during basal activity and stimulation. (C) Loss of EAAT2a function leads to neuronal hypoactivity during basal periods, likely reflecting reduced levels of available glutamate/glutamine. (D) Once glutamate is released in higher quantities from the presynapse (e.g. following light stimuli or spontaneous glutamate release), astroglia cannot sufficiently take up glutamate. Accumulated glutamate in the synaptic cleft hyperexcites postsynaptic neurons, which can lead to a cascade of glutamate release across the nervous system and results in epileptic seizures. Such recurrent epileptic seizures could also potentially lead to desensitization or a negative feedback to the presynapse $(\mathbf{C})$, which can further explain reduced basal neuronal activity in eaat $2 a^{-/}$mutants. 
due to depletion of the presynaptic glutamate pool after seizures in combination with impaired glutamate recycling. Since astroglial uptake of glutamate is impaired in eaat $2 a^{-/}$mutants, the conversion of glutamate to glutamine is likely reduced, resulting in an impaired glutamateglutamine cycle ${ }^{44}$. This eventually leads to lesser glutamate reconversion in the presynaptic neurons, leading to hypoactivity ${ }^{45}$. Alternatively, impaired glutamate clearance from the synaptic cleft can lead to desensitization or reduction of postsynaptic glutamate receptors, especially ionotropic AMPA receptors ${ }^{46,47}$. Consequently, the sensitivity to basal extracellular glutamate fluctuations may be decreased in these animals, and only higher levels of extracellular glutamate induced by sensory stimulation lead to the observed hyperexcitability. A third possibility is that the spontaneous release of glutamate from the presynapse may be suppressed by a negative feedback by neuropeptide modulators. As such, neuropeptide Y, which indirectly inhibits presynaptic glutamate release, has been shown to be upregulated in patients with resistant epilepsy and rodent models ${ }^{48,49}$. It is likely that all these mechanisms contribute to the switch between the hypoactive inter-ictal state and epileptic seizures. In fact, not only the recurrent spontaneous seizures of eaat $2 a^{-/-}$mutants, but also the reduced interictal brain activity mirror observations in human patients; de novo mutations in the human orthologue EAAT2 cause lower residual brain activity and profound intellectual disability ${ }^{18,19}$

In recent years, pharmacological and genetic zebrafish models have been used to advance the understanding of epileptogenesis ${ }^{28-30,50-57}$. Many of these studies focus on the inhibitory system by either pharmacologically or genetically manipulating GABA receptors. However, a better understanding of the major excitatory neurotransmitter system is of great importance, also considering that glutamate is the primary precursor of GABA ${ }^{44}$. In our model of targeting the glutamate transporter EAAT2a, we found several similarities to existing zebrafish models. Considering seizure propagation, we found that seizures in eaat $2 a^{-/-}$mutants initiate in midbrain and hindbrain regions that include important primary processing areas such as the optic tectum (homologous to mammalian superior colliculus ${ }^{58}$ ) and the cerebellum ${ }^{59}$. Interestingly, the anterior forebrain (homologous to mammalian neocortex ${ }^{60}$ ) is recruited only with a significant delay. Compared to the PTZ-induced model leading to a lack of neuronal inhibition, our results are similar to findings of one study ${ }^{10}$, yet contradicting another study showing epileptic propagation from anterior to posterior brain regions ${ }^{29}$. However, both studies found neuronal microcircuits to be a crucial step in seizure propagation. In line with this idea, we observed not only global but also localized seizures in eaat $2 a^{-/-}$ 10 mutants, all starting in subcortical regions. These findings support the hypothesis that highly connected hub-like regions play an important role as gate keepers between seizure foci and global brain networks ${ }^{61}$. Furthermore, it suggests that the recruitment of seizure-prone (ictogenic) hubs during seizure propagation might be more crucial than the initial dysfunction itself. It is likely that this recruitment of specific circuits is dependent on the current brain state, which has been suggested to influence seizure probability ${ }^{62}$. In fact, eaat $2 a^{-1}$ animals suffering global reflex seizures also always exhibited spontaneous global seizures. Hence, future studies in our model may help to understand the transition from local to global seizure networks, and the role of astroglia in these transitions.

We also observed fundamental differences between our eaat $2 a^{-/}$mutants and existing zebrafish models. Our model reflects that epilepsy is more than just a seizure disorder. In fact, subsequent neurobiological and cognitive consequences is part of the epilepsy definition ${ }^{9}$. While existing zebrafish models helped to observe several aspects of epileptic hyperexcitability ${ }^{10,29,30,50}$, our eaat $2 a^{-/}$mutants display reduced inter-ictal brain activity. The low neuronal and astroglial network activity in eaat $2 a^{-/}$mutants likely reflects pathological changes in the brain ${ }^{40}$, potentially corresponding to intellectual disabilities in patients with EAAT2 de novo mutations ${ }^{18,19}$. We also observed decreased brain size in eaat $2 a^{-/}$larvae, possibly reflecting cerebral atrophy found in patients with EAAT2 mutations ${ }^{18,19}$. Furthermore, our findings on overall reduced locomotion in eaat $2 a^{-/}$zebrafish is consistent with reduced muscle tone in human patients ${ }^{18,19}$. Hence, relying solely on increased locomotion as seizure readout in zebrafish models may miss important aspects of epilepsy. Accordingly, focusing not only on the seizure-related hyperactivity but also on the pathophysiology of reduced inter-ictal activity might help unraveling important underlying details of the combined clinical presentation of DEE. Our findings in eaat $2 a^{-/}$mutant zebrafish may also have a broader relevance for epilepsy research. The current clinical dichotomy between focal and generalized seizures is operational and may not reflect mechanistic distinctions ${ }^{63-65}$. Recent research in human patients using advanced neurophysiological methods and functional imaging is transforming our understanding on ictogenesis. Even the archetypical 'generalized' absence seizures involve rather selective parts of the brain, and not the entire cortex as suggested by conventional scalp electroencephalogram ${ }^{65,66}$. Our transparent zebrafish model enables detailed whole-brain imaging of widespread glianeuron networks ${ }^{67,68}$. We observed at high temporal resolution a local subcortical seizure origin and subsequent global propagation. It has been proposed that all seizures in fact initiate within local networks, and subsequent spreading results from a lost balance be- 
tween local and global network connectivity ${ }^{69,70}$. Subcortical networks are likely of particular interest, given that they have been shown to be intimately involved in seizures traditionally thought to arise from cortical lobes ${ }^{71,72}$. Furthermore, the eaat $2 a^{-/}$model enables a certain temporal control of seizure onset through light-induction, providing a prominent window to study ictogenesis. Future studies in our eaat $2 a^{-/}$model may help to improve the understanding on interactions between local and global seizure networks. For all these reasons, we propose to use our novel epilepsy model comprehensively to further the understanding of underlying epileptogenic mechanisms. We argue that our astroglial eaat $2 a^{-/}$mutant zebrafish model will provide an unexplored platform for identifying new treatment approaches, especially taking into account gliotransmission as a promising novel target ${ }^{73-76}$.

\section{METHODS}

\section{Fish husbandry}

Zebrafish (Danio rerio) were kept under standard conditions ${ }^{77}$. In this study, WIK and Tübingen wildtype strains were used. For calcium imaging experiments, eaat $2 a^{+/-}$mutant animals were outcrossed with Tg(elavl3:GCaMP5G); nacre $^{-/ 2} 78$ (epifluorescence microscope), $\operatorname{Tg}$ (gfap:Gal4)nw7;Tg(UAS:GCaMP6s) 10,79 fish (two-photon spontaneous recordings) and Tg(elavl3:GCaMP6s) ${ }^{17}$ (two-photon light-stimulation recordings). For experiments, adult eaat $2 a^{+/}$animals were set up pairwise and embryos were raised in $\mathrm{E} 3$ medium $(5 \mathrm{mM} \mathrm{NaCl}, 0.17 \mathrm{mM} \mathrm{KCl}, 0.33 \mathrm{mM}$ $\mathrm{CaCl} 2,0.33 \mathrm{mM} \mathrm{MgSO} 4,10^{-5} \%$ methylene blue) (Zürich) or in egg water $\left(60 \mathrm{mg} / 1 \mathrm{marine}\right.$ salt, $10^{-4} \%$ methylene blue) (Trondheim) at $28^{\circ} \mathrm{C}$. As control, eaat $2 a^{-/}$larvae were compared to eaat $2 a^{+/+}$and eaat $2 a^{+/-}$siblings. All experiments were conducted in accordance with local authorities (Zürich Switzerland: Kantonales Veterinäramt TV4206, Trondheim Norway: directive 2010/63/EU of the European Parliament and the Council of the European Union and the Norwegian Food Safety Authorities).

\section{In situ hybridization}

eaat2a (ENSDARG00000102453) cloning into the TOPO pCRII vector (TA Cloning Kit Dual Promoter, Invitrogen, Basel, Switzerland) and preparation of digoxigenin (DIG)-labeled antisense RNA probes is described elsewhere ${ }^{15,16}$. RNA probes were applied on whole-mount zebrafish larvae (5 dpf) and adult brain cross sections at a concentration of $2 \mathrm{ng} / \mu \mathrm{L}$ at $64^{\circ} \mathrm{C}$ overnight ${ }^{80}$. Stained and paraformaldehyde (PFA) post-fixed embryos (in glycerol) and sections were imaged with an Olympus BX61 brightfield microscope. Images were adjusted for brightness and contrast using Affinity Photo Version 1.8 and assembled Affinity Designer Version 1.7.

\section{Immunohistochemistry}

Generation of the chicken anti-EAAT2a (zebrafish) antibody is described elsewhere ${ }^{16} .5 \mathrm{dpf}$ larvae were fixed in 4\% PFA in PBS ( $\mathrm{pH} 7.4$ ) at room temperature for 40 minutes. Embryos were cryo-protected in 30\% sucrose in PBS at $4^{\circ} \mathrm{C}$ overnight, embedded in Tissue Freezing Medium TFMTM (Electron Microscopy Sciences), cryo-sectioned at 14-16 $\mu \mathrm{m}$ and mounted onto Superfrost slides (Thermo Fisher Scientific). Immunohistochemistry was performed as described before ${ }^{16}$. Chicken anti-EAAT2a 1:500, mouse antisynaptic vesicle 2 (IgG1, 1:100, DSHB USA), mouse anti-acetylated tubulin (IgG2b, 1:500, Sigma 7451) and mouse anti-glutamine synthetase (IgG2a, 1:200, EMD Millipore, MAB302) were used as primary antibodies. Secondary antibodies were goat antichicken Alexa Fluor 488, goat anti-mouse IgG2a Alexa Fluor 568, goat anti-mouse IgG2b Alexa Fluor 647 and goat anti-mouse IgG1 Alexa Fluor 647, all 1:500 (all from Invitrogen, Thermo Fisher Scientific). Slides were cover-slipped using Mowiol (Polysciences) containing DABCO (Sigma-Aldrich) and imaged with a TCS LSI confocal microscope (Leica Microsystems). Images were adjusted for brightness and contrast using Affinity Photo Version 1.8 and assembled Affinity Designer Version 1.7.

\section{CRISPR/Cas9-mediated mutagenesis and geno- typing \\ CRISPR target sites for eaat2a} (ENSDARG00000102453) of the 5'-GG(N18)NGG3' motif favorable for T7-mediated in vitro transcription ${ }^{81}$ were selected using the https:// chopchop.cbu.uib.no/ and www.zifit.partners.org prediction tools. Synthesis of single guide RNA (sgRNA) was performed using a PCR based approach as follows. dsDNA of the target region was amplified with a high fidelity Phusion polymerase (New England Bio Labs) using the forward primer $\mathrm{sg} 1$ together with the common reverse primer sg2 (Supplementary Table 1). sgRNA was T7 in vitro transcribed (MEGAshortscriptTM T7 Transcription Kit, Ambion) and subsequently purified using the MEGAclearTM Kit (Ambion).

The injection mix consisting of $160 \mathrm{ng} / \mu \mathrm{L}$ sgRNA, 1 $\mu \mathrm{g} / \mu \mathrm{L}$ Cas 9 protein (Flag/ or GFP-tagged Cas9 kindly provided by Prof. Dr. C. Mosimann and Prof. Dr. M. Jineck) and $300 \mathrm{mM} \mathrm{KCl}$ was incubated at $37^{\circ} \mathrm{C}$ for 10 minutes to enable Cas $9 /$ sgRNA complex formation. One-cell staged embryos were injected with $1 \mathrm{~nL}$ injection mix into the cell. Mutation rate efficiency was tested by genotyping a pool of around 10-15 injected F0 larvae per clutch while their siblings were raised to adulthood. Adult F0 crispant fish were outcrossed to Tübingen wild-type animals. F1 embryos were genotyped at 3 dpf by larval tail biopsies ${ }^{82}$ and raised in single tanks. The eaat $2 a$ target site was PCR amplified with a fast-cycling polymerase (KAPA2G Fast HotStart PCR kit, KAPA 
Biosystems) (primers: sense (fw) and antisense (rev, Supplementary Table 1)). Amplicons were cloned into pCR 2.1-TOPO vectors (Invitrogen) and sequenced. The resulting heterozygous mutant line carrying one copy of a -13 null allele was repeatedly outcrossed to wild-type and transgenic fish to generate stable heterozygous F2, F3 and F4 generations. eaat $2 a$ mutant fish can be genotyped by the above-mentioned PCR amplification and a subsequent gel-electrophoresis, which allows detection of the 13 base pair deletion. Larvae were genotyped after each experiment. After two-photon microscopy, all larvae were genotyped by means of a PCR melting curve analysis using SYBR Green (PowerUp SYBR Green Master Mix, Thermo Fisher Scientific). For qRT-PCR experiments, wild-type progeny of eaat $2 a^{+/}$incrosses were selected by pregenotyping using the Zebrafish Embryo Genotyper (ZEG, wFluidx) as described previously ${ }^{83}$. Briefly, 34 dpf embryos were loaded individually onto the ZEG chip in $12 \mu \mathrm{LE} 3$ and vibrated for 10 minutes at 1.4 Volts. Subsequently, $8 \mu \mathrm{L}$ of each sample E3 was used directly for PCR as described above (KAPA Biosystems). Embryos were kept in 48-well plates until genotyping was achieved.

\section{Zebrafish survival analysis and length measure- ments}

Embryos were raised separately in 24-well plates containing $1.5 \mathrm{~mL}$ E3 medium, which was changed daily. From 5 dpf on, larvae were fed manually. Animal survival was monitored daily over an 11-day period. From 1 to $7 \mathrm{dpf}$, larvae were anaesthetized by tricaine $(0.2 \mathrm{mg} / \mathrm{mL})$ and individually imaged with an Olympus MVX10 microscope. Body lengths were measured along the spinal cord using Fiji ImageJ (National Institutes of Health) and further analyzed using R software version 3.6.0 with the RStudio version 1.2.1335 interface ${ }^{84,85}$.

\section{Behavioral analysis}

Swimming patterns of $5 \mathrm{dpf}$ larvae were recorded using the ZebraBox system (ViewPoint Life Sciences). The room was kept at $27^{\circ} \mathrm{C}$ throughout the recordings. Larvae were individually placed at randomized positions of 48-well plates containing $1 \mathrm{~mL} \mathrm{E3}$ medium and transferred into the recording chamber for a minimum of 10 minutes acclimatization. Subsequently, recordings of 30 minutes in normal light conditions (20\% light intensity) were performed. An automated camera tracked individual larvae (threshold black 30) and detected the distance and duration moved by each larva exceeding $1 \mathrm{~mm}$ per second in accumulating five-second intervals. Data processing and analysis was done with a custom code written in $\mathrm{R}$ with the RStudio interface ${ }^{84,85}$. Software-related artefacts were removed in a blinded manner. Velocity of long-lasting bursts were calculated as distance di- vided by time for every five-second interval in which the animal moved at least 3.5 seconds.

\section{Combined LFP experiments together with epifluo- rescence iGluSnFR imaging}

Simultaneous LFP (local field potential) recordings of seizure activity and epifluorescence imaging of $i G l u S n F R$ signals were performed in $5 \mathrm{dpf}$ $T g$ (gfap:iGluSnFR) zebrafish larvae ${ }^{10}$. First, zebrafish larvae were anesthetized in $0.02 \%$ MS222 and paralyzed by $\alpha$-bungarotoxin injection ${ }^{86}$. Next, the larvae were embedded in $1-1.5 \%$ low melting point agarose (Fisher Scientific) in a recording chamber (Fluorodish, World Precision Instruments). Artificial fish water (AFW, $1.2 \mathrm{~g}$ marine salt in $20 \mathrm{~L}$ RO water) was constantly perfused during the experiments. For LFP recordings, a borosilicate glass patch clamp pipette (9-15 MOhms) loaded with teleost artificial cerebrospinal fluid ${ }^{87}$ (ACSF, containing in mM: $123.9 \mathrm{NaCl}, 22 \mathrm{D}$-glucose, $2 \mathrm{KCl}$, $1.6 \mathrm{MgSO}_{4} 7 \mathrm{H}_{2} \mathrm{O}, 1.3 \mathrm{KH}_{2} \mathrm{PO}_{4}, 24 \mathrm{NaHCO}_{3}, 2 \mathrm{CaCl}_{2}$ $2 \mathrm{H}_{2} \mathrm{O}$ ) was inserted in the forebrain ${ }^{75}$. LFP recordings were performed by a MultiClamp 700B amplifier, in current clamp mode at $10 \mathrm{kHz}$ sampling rate, and band pass filtered at $0.1-1000 \mathrm{~Hz}$. For imaging $i G l u S n F R$ signals, microscopy images were collected at $10 \mathrm{~Hz}$ sampling rate, using a Teledyne QImaging QI825 camera, in combination with Olympus BX51 fluorescence microscope and Olympus UMPLANFL 10X water immersion objective. Data acquisition of LFP signals were done in MATLAB (Mathworks), and iGluSnFR signals were done in Ocular Image Acquisition Software. All data were analyzed using MATLAB.

\section{Quantitative reverse transcription PCR}

5 dpf larvae were anesthetized on ice and brains dissected using an insect pin and a syringe needle in a dish containing RNA later (Sigma-Aldrich). Total RNA of equal pools of larval brains was extracted using the ReliaPrep kit (Promega). RNA was reverse transcribed to cDNA using the Super Script III Firststrand synthesis system (Invitrogen) using 1:1 ratio of random hexamers and oligo (dt) primers.

The qPCR reactions were performed using SsoAdvanced Universal SYBR Green Supermix on a CFX96 Touch Real-Time PCR Detection System (Bio-rad). Primer (Supplementary Table 2) efficiencies were calculated by carrying out a dilution series. After the primer efficiencies were determined equal, brain samples were used for qPCR using $10 \mathrm{ng}$ of cDNA per reaction. The controls "no reverse transcription control" (nRT control) and "no template control" (NTC) were performed with every qPCR reaction. g6pd and $b 2 m$ were chosen as reference genes. All reactions were performed in technical triplicates. Data was analyzed in CFX Maestro Software from Bio-Rad and Microsoft Excel. Statistical analysis was performed between $\mathrm{dCt}$ values. 


\section{Calcium imaging and data analysis}

\section{a. Epifluorescence microscope}

5 dpf larvae in the $\operatorname{Tg}$ (elavl3:GCaMP5G) background were individually immobilized in a drop of $1.8 \%$ NuSieveTM GTGTM low melting temperature agarose (Lonza) with the tail freed from agarose in a small cell culture dish (Corning Incorporated) filled with water. Calcium signals were recorded using an Olympus BX51 WI epifluorescence microscope and by means of a camera ( $4 \mathrm{~Hz}$ sampling rate) and the VisiView software established by the Visitron Systems GmbH.

In each experiment, the GCaMP5G fluorescence signal of manually selected ROIs (whole brain, forebrain, midbrain and hindbrain) was extracted using Fiji ImageJ (National Institutes of Health). For each time point, the mean intensity of each ROI was measured and further processed using a custom script in $\mathrm{R}$ with the RStudio interface ${ }^{84,85}$. The baseline $\left(\mathrm{F}_{0}\right)$ of every ROI was calculated as $1^{\text {st }}$ percentile of the entire fluorescence trace per fish. Subsequently, the fractional change in fluorescence $\left(\Delta \mathrm{F} / \mathrm{F}_{0}\right)$ of each ROI was computed to normalize the values obtained. In order to compare basal activity between eaat $2 a^{-/}$, eaat $2 a^{+/-}$and eaat $2 a^{+/+}$larvae, the standard deviation of F0 for the whole brain was calculated and averaged over five two-minute time windows per animal (same random windows for all fish, but adjusted if during seizure). Seizure duration in eaat $2 a^{-/}$mutants was defined as the period from first time point where $\Delta \mathrm{F} / \mathrm{F}$ is greater than $50 \%$ (seizure initiation) until first time point where $\Delta \mathrm{F} / \mathrm{F}$ is below $50 \%$ in the midbrain.

\section{b. Two-photon microscope}

Two-photon calcium recordings were performed on 5 dpf eaat $2 a$ mutant $\operatorname{Tg}$ (gfap:Gal4)nw7; $\operatorname{Tg}(U A S: G$ CaMP6s) zebrafish ${ }^{10}$. Larvae were pre-selected based on morphological phenotype, paralyzed by injecting $1 \mathrm{~nL}$ of $\alpha$-bungarotoxin (Invitrogen BI601, $1 \mathrm{mg} / \mathrm{mL}$ ) into the spinal cord, embedded in 1.5-2\% low melting point agarose (LMP, Fisher Scientific) in a recording chamber (Fluorodish, World Precision Instruments) and constantly perfused with artificial fish water (AFW, $60 \mathrm{mg} / 1$ marine salt in RO water) during imaging. The cerebral blood flow was assessed in the prosencephalic arteries or anterior cerebral veins ${ }^{88}$ before and after the experiment, and animals without cerebral blood flow were excluded from the final analysis. Imaging was performed in a two-photon microscope (Thorlabs Inc and Scientifica Inc) with a x16 water immersion objective (Nikon, NA 0.8, LWD 3.0, plan). Excitation was achieved by a mode-locked Ti:Sapphire laser (MaiTai Spectra-Physics) tuned to $920 \mathrm{~nm}$. Single plane recordings of 1536 x 650 pixels were obtained at an acquisition rate of $24 \mathrm{~Hz}$. The plane was oriented towards the glial cells in the boundary region between the telencephalon (anterior forebrain) and the anterior thalamus (posterior forebrain). First, spontaneous calcium activity was measured for 60 minutes. Subsequently for a subgroup of the fish, a custom-made Arduino was used to apply two 10-second red-light stimuli $(625 \mathrm{~nm})$. Light was flashed after 5 and 10 minutes of the total duration of 15 minutes.

Images were aligned, cells detected and $\Delta \mathrm{F} / \mathrm{F} 0$ relative to the baseline calculated using the algorithm previously described ${ }^{89,90}$, with adaptions as follows. Glial cells along the ventricle were semi-automatically detected and subsequently assigned manually according to their location ${ }^{10,89,91}$. Baseline was computed within a moving window of 80 seconds as the $8^{\text {th }}$ percentile of activity defining noise with a Gaussian curve fit using an adapted algorithm ${ }^{92,93}$. Resampled $\Delta \mathrm{F} / \mathrm{F} 0$ traces of $4 \mathrm{~Hz}$ were used to detect glial calcium signals. Calcium events were identified by detecting events significantly different from noise level within a $95 \%$ confidence interval ${ }^{92,93}$. For spontaneous recordings, glial activity was quantified in the following two-minute time windows: basal (between seizure activity, averaged over six time points per fish), preictal (preceding seizure onset) and ictal (during seizure). Seizure onset frames were defined as time points where the averaged $\Delta \mathrm{F} / \mathrm{F} 0$ of all cells not located along the ventricles reached 50. For light stimuli recordings, glial activity was compared between one minute prior to and one minute following light stimuli. In each period, a cell was considered active if at least one event occurred. The overall activity of active cells was calculated by the trapezoidal numerical integration method (function trapz, MAT$\mathrm{LAB}$ ) to get the area under the curve (AUC). The amplitude of each event was determined as its respective maximum peak.

Light stimulus assay of neuronal activity was performed on 5 dpf eaat $2 a^{+/} \operatorname{Tg}$ (elavl3:GCaMP6s) incross progenies. Mutant larvae were pre-selected, paralyzed and embedded as described above. After the agarose solidified for 10 minutes, AFW was added as an immersion medium before 20 more minutes of agarose settling. The animals were acclimatized in the setup for 20 minutes before volumetric recordings of ten planes of $1536 \times 650$ pixels were obtained using a Piezo element at a rate of 2,43 Hz. Excitation was achieved as described above. Baseline spontaneous calcium activity was recorded for ten minutes in darkness, followed by a red-light stimulus train using a red LED (LZ1-00R105, LedEngin; 625$\mathrm{nm})$ placed in front of the animal. Five light stimuli with five-minute inter-stimulus intervals were applied by an Arduino-device. Images were aligned as described above. Regions of interest (ROIs) were manually drawn on one single plane of maximal information. F0 baseline was computed as $1^{\text {st }}$ percentile of the entire fluorescence trace per ROI. 


\section{Statistical analysis}

Statistical analysis was done using R software version 3.6.0 with the RStudio version 1.2.1335 interface $^{84,85}$. Data sets were tested for normality using quantile-quantile plots and Shapiro-Wilk test. Normally distributed data was analyzed using Welch two-sample unpaired t-test (Fig. $5 \mathrm{i}$ and $\mathrm{m}$ ). Wilcoxon rank-sum test was used for non-paired analysis (Fig. 2 g, Fig. 5g, h, j-1), Wilcoxon signed rank test for paired analysis (Fig. 5e, f) and two-sample Kolmogorov-Smirnov test for equality between distributions (Fig. 3f). Kruskal-Wallis rank-sum test with Wilcoxon rank-sum posthoc test was used for nonpaired analysis between all three genotypes (Fig. 2b, Fig. 3c, Fig. 4j). $P<0.05$ was considered as statistically significant.

\section{Data and Code availability}

The datasets and codes supporting this study have not been deposited in a public repository, but are available from the corresponding authors upon request.

\section{REFERENCES}

1. Allen, N. J. \& Eroglu, C. Cell Biology of Astrocyte-Synapse Interactions. Neuron 96, 697708; 10.1016/j.neuron.2017.09.056 (2017).

2. Clarke, L. E. \& Barres, B. A. Emerging roles of astrocytes in neural circuit development. Nature reviews. Neuroscience 14, 311-321; 10.1038/ nrn3484 (2013).

3. Bazargani, N. \& Attwell, D. Astrocyte calcium signaling. The third wave. Nature neuroscience 19, 182-189; 10.1038/nn.4201 (2016).

4. Santello, M., Toni, N. \& Volterra, A. Astrocyte function from information processing to cognition and cognitive impairment. Nature neuroscience 22, 154-166; 10.1038/s41593-0180325-8 (2019).

5. Danbolt, N. C. Glutamate uptake. Progress in Neurobiology 65, 1-105 (2001).

6. Coulter, D. A. \& Eid, T. Astrocytic regulation of glutamate homeostasis in epilepsy. Glia 60, 1215-1226; 10.1002/glia.22341 (2012).

7. Rothstein, J. D. et al. Knockout of Glutamate Transporters Reveals a Major Role for Astroglial Transport in Excitotoxicity and Clearance of Glutamate. Neuron 16, 675-686; 10.1016/S08966273(00)80086-0 (1996).

8. Vandenberg, R. J. \& Ryan, R. M. Mechanisms of glutamate transport. Physiological reviews 93, 1621-1657; 10.1152/physrev.00007.2013 (2013).

9. Fisher, R. S. et al. ILAE official report: a practical clinical definition of epilepsy. Epilepsia 55, 475482; 10.1111/epi.12550 (2014).

10. Diaz Verdugo, C. et al. Glia-neuron interactions underlie state transitions to generalized seizures. Nat Commun 10, 475; 10.1038/s41467-01911739-z (2019).
11. Devinsky, O., Vezzani, A., Najjar, S., Lanerolle, N. C. de \& Rogawski, M. A. Glia and epilepsy: excitability and inflammation. Trends in neurosciences $\quad 36, \quad 174-184 ; \quad 10.1016 /$ j.tins.2012.11.008 (2013).

12. Steinhäuser, C., Seifert, G. \& Bedner, P. Astrocyte dysfunction in temporal lobe epilepsy: $\mathrm{K}+$ channels and gap junction coupling. Glia 60, 1192-1202; 10.1002/glia.22313 (2012).

13. Rosch, R. E. \& Dulla, C. G. A Tale of two Networks-Glial Contributions to Generalized Seizures. Epilepsy currents 20, 108-110; 10.1177/1535759720906115 (2020).

14. Eid, T., Lee, T.-S. W., Patrylo, P. \& Zaveri, H. P. Astrocytes and Glutamine Synthetase in Epileptogenesis. Journal of neuroscience research 97, 1345-1362; 10.1002/jnr.24267 (2019).

15. Gesemann, M., Lesslauer, A., Maurer, C. M., Schonthaler, H. B. \& Neuhauss, S. C. F. Phylogenetic analysis of the vertebrate excitatory/ neutral amino acid transporter (SLC1/EAAT) family reveals lineage specific subfamilies. $B M C$ evolutionary biology 10, 117; 10.1186/14712148-10-117 (2010).

16. Niklaus, S. et al. Shaping of Signal Transmission at the Photoreceptor Synapse by EAAT2 Glutamate Transporters. eNeuro 4; 10.1523/ ENEURO.0339-16.2017 (2017).

17. Vladimirov, N. et al. Light-sheet functional imaging in fictively behaving zebrafish. Nature methods 11, 883-884; 10.1038/nmeth.3040 (2014).

18. Epi4K Consortium. De Novo Mutations in SLC1A2 and CACNA1A Are Important Causes of Epileptic Encephalopathies. American journal of human genetics 99, 287-298; 10.1016/ j.ajhg.2016.06.003 (2016).

19. Guella, I. et al. De Novo Mutations in YWHAG Cause Early-Onset Epilepsy. American journal of human genetics 101, 300-310; 10.1016/ j.ajhg.2017.07.004 (2017).

20. Jurisch-Yaksi, N., Yaksi, E. \& Kizil, C. Radial glia in the zebrafish brain: Functional, structural, and physiological comparison with the mammalian glia. Glia; 10.1002/glia.23849 (2020).

21. Mu, Y. et al. Glia Accumulate Evidence that Actions Are Futile and Suppress Unsuccessful Behavior. Cell 178, 27-43.e19; 10.1016/ j.cell.2019.05.050 (2019).

22. Vandenberg, R. J. \& Ryan, R. M. Mechanisms of glutamate transport. Physiological reviews 93, 1621-1657; 10.1152/physrev.00007.2013 (2013).

23. Yaksi, E., Jamali, A., Diaz Verdugo, C. \& JurischYaksi, N. Past, present and future of zebrafish in epilepsy research. The FEBS journal; 10.1111/ febs.15694 (2021). 
24. Hortopan, G. A., Dinday, M. T. \& Baraban, S. C. Zebrafish as a model for studying genetic aspects of epilepsy. Disease models \& mechanisms 3, 144-148; 10.1242/dmm.002139 (2010).

25. Gawel, K. et al. Seizing the moment: Zebrafish epilepsy models. Neuroscience and biobehavioral reviews 116, 1-20; 10.1016/ j.neubiorev.2020.06.010 (2020).

26. Budick, S. A. \& O'Malley, D. M. Locomotor repertoire of the larval zebrafish: swimming, turning and prey capture. The Journal of experimental biology 203, 2565-2579 (2000).

27. Kalueff, A. V. et al. Towards a comprehensive catalog of zebrafish behavior 1.0 and beyond. Zebrafish 10, 70-86; 10.1089/zeb.2012.0861 (2013).

28. Baraban, S. C., Taylor, M. R., Castro, P. A. \& Baier, H. Pentylenetetrazole induced changes in zebrafish behavior, neural activity and c-fos expression. Neuroscience 131, 759-768; 10.1016/ j.neuroscience.2004.11.031 (2005).

29. Liu, J. \& Baraban, S. C. Network Properties Revealed during Multi-Scale Calcium Imaging of Seizure Activity in Zebrafish. eNeuro 6; 10.1523/ ENEURO.0041-19.2019 (2019).

30. Turrini, L. et al. Optical mapping of neuronal activity during seizures in zebrafish. Scientific reports 7, 3025; 10.1038/s41598-017-03087-z (2017).

31. Hanson, E. et al. Tonic Activation of GluN2C/ GluN2D-Containing NMDA Receptors by Ambient Glutamate Facilitates Cortical Interneuron Maturation. The Journal of neuroscience : the official journal of the Society for Neuroscience 39, 3611-3626; 10.1523/ JNEUROSCI.1392-18.2019 (2019).

32. MacDonald, R. B., Kashikar, N. D., Lagnado, L. \& Harris, W. A. A Novel Tool to Measure Extracellular Glutamate in the Zebrafish Nervous System In Vivo. Zebrafish; 10.1089/ zeb.2016.1385 (2016).

33. Koepp, M. J., Caciagli, L., Pressler, R. M., Lehnertz, K. \& Beniczky, S. Reflex seizures, traits, and epilepsies: from physiology to pathology. The Lancet Neurology 15, 92-105; 10.1016/S1474-4422(15)00219-7 (2016).

34. Haug, M. F., Gesemann, M., Mueller, T. \& Neuhauss, S. C. F. Phylogeny and expression divergence of metabotropic glutamate receptor genes in the brain of zebrafish (Danio rerio). The Journal of comparative neurology 521, 15331560; 10.1002/cne.23240 (2013).

35. Tanaka, K. et al. Epilepsy and exacerbation of brain injury in mice lacking the glutamate transporter GLT-1. Science (New York, N.Y.) 276, 1699-1702 (1997).

36. Wagner, M. et al. Biallelic Mutations in SLC1A2; an Additional Mode of Inheritance for SLC1A2-
Related Epilepsy. Neuropediatrics 49, 59-62; 10.1055/s-0037-1606370 (2018).

37. Allen, A. S. et al. De novo mutations in epileptic encephalopathies. Nature 501, 217-221; 10.1038/ nature12439 (2013).

38. Petr, G. T. et al. Conditional deletion of the glutamate transporter GLT-1 reveals that astrocytic GLT-1 protects against fatal epilepsy while neuronal GLT-1 contributes significantly to glutamate uptake into synaptosomes. The Journal of neuroscience : the official journal of the Society for Neuroscience 35, 5187-5201; 10.1523/JNEUROSCI.4255-14.2015 (2015).

39. Stergachis, A. B. et al. Recurrent SLC1A2 variants cause epilepsy via a dominant negative mechanism. Annals of neurology; 10.1002/ ana.25477 (2019).

40. Scheffer, I. E. et al. ILAE classification of the epilepsies: Position paper of the ILAE Commission for Classification and Terminology. Epilepsia 58, 512-521; 10.1111/epi.13709 (2017).

41. Niklaus, S. \& Neuhauss, S. C. F. Genetic approaches to retinal research in zebrafish. Journal of neurogenetics, 1-18; 10.1080/01677063.2017.1343316 (2017).

42. Seifert, G. \& Steinhäuser, C. Neuron-astrocyte signaling and epilepsy. Experimental neurology 244, 4-10; 10.1016/j.expneurol.2011.08.024 (2013).

43. Boison, D. \& Steinhäuser, C. Epilepsy and astrocyte energy metabolism. Glia 66, 12351243; 10.1002/glia.23247 (2018).

44. Bak, L. K., Schousboe, A. \& Waagepetersen, H. $\mathrm{S}$. The glutamate/GABA-glutamine cycle: aspects of transport, neurotransmitter homeostasis and ammonia transfer. Journal of neurochemistry $\mathbf{9 8 ,}$ 641-653; 10.1111/j.1471-4159.2006.03913.x (2006).

45. Tani, H. et al. A local glutamate-glutamine cycle sustains synaptic excitatory transmitter release. Neuron 81, 888-900; 10.1016/ j.neuron.2013.12.026 (2014).

46. Trussell, L. O. \& Fischbach, G. D. Glutamate receptor desensitization and its role in synaptic transmission. Neuron 3, 209-218; 10.1016/08966273(89)90034-2 (1989).

47. Jayakar, S. S. \& Dikshit, M. AMPA receptor regulation mechanisms: future target for safer neuroprotective drugs. The International journal of neuroscience 114, 695-734; 10.1080/00207450490430453 (2004).

48. Tu, B., Timofeeva, O., Jiao, Y. \& Nadler, J. V. Spontaneous release of neuropeptide Y tonically inhibits recurrent mossy fiber synaptic transmission in epileptic brain. The Journal of neuroscience : the official journal of the Society for Neuroscience 25, 1718-1729; 10.1523/ JNEUROSCI.4835-04.2005 (2005). 
49. Furtinger, S. et al. Plasticity of Y1 and Y2 receptors and neuropeptide $\mathrm{Y}$ fibers in patients with temporal lobe epilepsy. The Journal of neuroscience : the official journal of the Society for Neuroscience 21, 5804-5812 (2001).

50. Samarut, É. et al. $\gamma$-Aminobutyric acid receptor alpha 1 subunit loss of function causes genetic generalized epilepsy by impairing inhibitory network neurodevelopment. Epilepsia 59, 20612074; 10.1111/epi.14576 (2018).

51. Liao, M. et al. Targeted knockout of GABA receptor gamma 2 subunit provokes transient light-induced reflex seizures in zebrafish larvae. Disease models \& mechanisms; 10.1242/ dmm.040782 (2019).

52. Tiraboschi, E. et al. New insights into the early mechanisms of epileptogenesis in a zebrafish model of Dravet syndrome. Epilepsia 61, 549560; 10.1111/epi.16456 (2020).

53. Ghannad-Rezaie, M., Eimon, P. M., Wu, Y. \& Yanik, M. F. Engineering brain activity patterns by neuromodulator polytherapy for treatment of disorders. Nat Commun 10, 2620; 10.1038/ s41467-019-10541-1 (2019).

54. Gawel, K. et al. Phenotypic Characterization of Larval Zebrafish (Danio rerio) with Partial Knockdown of the cacnala Gene. Molecular neurobiology 57, 1904-1916; 10.1007/s12035019-01860-x (2020).

55. Afrikanova, T. et al. Validation of the zebrafish pentylenetetrazol seizure model: locomotor versus electrographic responses to antiepileptic drugs. PloS one 8, e54166; 10.1371/ journal.pone.0054166 (2013).

56. Sourbron, J. et al. Drug repurposing for Dravet syndrome in scn1Lab-/- mutant zebrafish. Epilepsia 60, e8-e13; 10.1111/epi.14647 (2019).

57. Ibhazehiebo, K. et al. A novel metabolism-based phenotypic drug discovery platform in zebrafish uncovers HDACs 1 and 3 as a potential combined anti-seizure drug target. Brain : a journal of neurology 141, 744-761; 10.1093/brain/awx364 (2018).

58. Mueller, T. What is the Thalamus in Zebrafish? Frontiers in neuroscience 6, 64; 10.3389/ fnins.2012.00064 (2012).

59. Kita, E. M., Scott, E. K. \& Goodhill, G. J. Topographic wiring of the retinotectal connection in zebrafish. Developmental neurobiology 75, 542-556; 10.1002/dneu.22256 (2015).

60. Ito, H. \& Yamamoto, N. Non-laminar cerebral cortex in teleost fishes? Biology letters 5, 117121; 10.1098/rsbl.2008.0397 (2009).

61. Paz, J. T. \& Huguenard, J. R. Microcircuits and their interactions in epilepsy: is the focus out of focus? Nature neuroscience 18, 351-359; 10.1038/nn.3950 (2015).

62. Staba, R. J. \& Worrell, G. A. What is the 16 importance of abnormal "background" activity in seizure generation? Advances in experimental medicine and biology 813, 43-54; 10.1007/97894-017-8914-1_3 (2014).

63. Fisher, R. S. et al. Operational classification of seizure types by the International League Against Epilepsy: Position Paper of the ILAE Commission for Classification and Terminology. Epilepsia 58, 522-530; 10.1111/epi.13670 (2017).

64. Fernandez-Baca Vaca, G. \& Park, J. T. Focal EEG abnormalities and focal ictal semiology in generalized epilepsy. Seizure 77, 7-14; 10.1016/ j.seizure.2019.12.013 (2020).

65. Wolf, P. \& Beniczky, S. Understanding ictogenesis in generalized epilepsies. Expert review of neurotherapeutics 14, 787-798; 10.1586/14737175.2014.925803 (2014).

66. Bai, X. et al. Dynamic time course of typical childhood absence seizures: EEG, behavior, and functional magnetic resonance imaging. The Journal of neuroscience : the official journal of the Society for Neuroscience 30, 5884-5893; 10.1523/JNEUROSCI.5101-09.2010 (2010).

67. Wyatt, C., Bartoszek, E. M. \& Yaksi, E. Methods for studying the zebrafish brain: past, present and future. The European journal of neuroscience 42, 1746-1763; 10.1111/ejn.12932 (2015).

68. Ahrens, M. B. et al. Brain-wide neuronal dynamics during motor adaptation in zebrafish. Nature 485, 471-477; 10.1038/nature11057 (2012).

69. Stam, C. J. Epilepsy: what can we learn from modern network theories. Epileptologie 33, 3843 (2016).

70. Blumenfeld, H. What is a seizure network? Longrange network consequences of focal seizures. Advances in experimental medicine and biology 813, 63-70; 10.1007/978-94-017-8914-1_5 (2014).

71. Picard, F. et al. Alteration of the in vivo nicotinic receptor density in ADNFLE patients: a PET study. Brain : a journal of neurology 129, 20472060; 10.1093/brain/awl156 (2006).

72. Motelow, J. E. et al. Decreased subcortical cholinergic arousal in focal seizures. Neuron 85, 561-572; 10.1016/j.neuron.2014.12.058 (2015).

73. Riquelme, J., Wellmann, M., Sotomayor-Zárate, R. \& Bonansco, C. Gliotransmission: A Novel Target for the Development of Antiseizure Drugs. The Neuroscientist : a review journal bringing neurobiology, neurology and psychiatry 26, 293309; 10.1177/1073858420901474 (2020).

74. Eimon, P. M. et al. Brain activity patterns in highthroughput electrophysiology screen predict both drug efficacies and side effects. Nat Commun 9, 219; 10.1038/s41467-017-02404-4 (2018).

75. Zhang, Y. et al. Pharmacological characterization of an antisense knockdown zebrafish model of Dravet syndrome: inhibition of epileptic seizures 
by the serotonin agonist fenfluramine. PloS one 10, e0125898; 10.1371/journal.pone.0125898 (2015).

76. Cunliffe, V. T. et al. Epilepsy research methods update: Understanding the causes of epileptic seizures and identifying new treatments using non-mammalian model organisms. Seizure 24, 44-51; 10.1016/j.seizure.2014.09.018 (2015).

77. Mullins, M. C., Hammerschmidt, M., Haffter, P. \& Nusslein-Volhard, C. Large-scale mutagenesis in the zebrafish: in search of genes controlling development in a vertebrate. Current biology : $C B$ 4, 189-202 (1994).

78. Akerboom, J. et al. Optimization of a GCaMP calcium indicator for neural activity imaging. The Journal of neuroscience : the official journal of the Society for Neuroscience 32, 13819-13840; 10.1523/JNEUROSCI.2601-12.2012 (2012).

79. Muto, A. et al. Activation of the hypothalamic feeding centre upon visual prey detection. Nat Commun 8, 15029; 10.1038/ncomms 15029 (2017).

80. Huang, Y. Y., Haug, M. F., Gesemann, M. \& Neuhauss, S. C. F. Novel expression patterns of metabotropic glutamate receptor 6 in the zebrafish nervous system, 2012.

81. Gagnon, J. A. et al. Efficient mutagenesis by Cas9 protein-mediated oligonucleotide insertion and large-scale assessment of single-guide RNAs. PloS one 9, e98186; 10.1371/ journal.pone.0098186 (2014).

82. Wilkinson, R. N., Elworthy, S., Ingham, P. W. \& van Eeden, Fredericus J M. A method for highthroughput PCR-based genotyping of larval zebrafish tail biopsies. BioTechniques 55, 314316; 10.2144/000114116 (2013).

83. Lambert, C. J. et al. An automated system for rapid cellular extraction from live zebrafish embryos and larvae: Development and application to genotyping. PloS one 13, e0193180; $\quad$ 10.1371/journal.pone.0193180 (2018).

84. R Core Team. R: A Language and Environment for Statistical Computing (R Foundation for Statistical Computing, Vienna, Austria, 2019).

85. RStudio Team. RStudio: Integrated Development for $R$ (RStudio, Inc., Boston, MA, 2018).

86. Olstad, E. W. et al. Ciliary Beating Compartmentalizes Cerebrospinal Fluid Flow in the Brain and Regulates Ventricular Development. Current biology : $C B$ 29, 229241.e6; 10.1016/j.cub.2018.11.059 (2019).

87. Mathieson, W. B. \& Maler, L. Morphological and electrophysiological properties of a novel in vitro preparation: the electrosensory lateral line lobe brain slice. Journal of comparative physiology. A, Sensory, neural, and behavioral physiology 163, 489-506; 10.1007/BF00604903 (1988).
88. Isogai, S., Horiguchi, M. \& Weinstein, B. M. The vascular anatomy of the developing zebrafish: an atlas of embryonic and early larval development. Developmental biology 230, 278-301; 10.1006/ dbio.2000.9995 (2001).

89. Reiten, I. et al. Motile-Cilia-Mediated Flow Improves Sensitivity and Temporal Resolution of Olfactory Computations. Current biology : $C B$ 27, 166-174; 10.1016/j.cub.2016.11.036 (2017).

90. Ohki, K., Chung, S., Ch'ng, Y. H., Kara, P. \& Reid, R. C. Functional imaging with cellular resolution reveals precise micro-architecture in visual cortex. Nature 433, 597-603; 10.1038/ nature03274 (2005).

91. Jetti, S. K., Vendrell-Llopis, N. \& Yaksi, E. Spontaneous activity governs olfactory representations in spatially organized habenular microcircuits. Current biology : $C B$ 24, 434-439; 10.1016/j.cub.2014.01.015 (2014).

92. Romano, S. A. et al. An integrated calcium imaging processing toolbox for the analysis of neuronal population dynamics. PLoS computational biology 13, e1005526; 10.1371/ journal.pcbi.1005526 (2017).

93. Fore, S. et al. Functional properties of habenular neurons are determined by developmental stage and sequential neurogenesis. Science advances $\mathbf{6}$; 10.1126/sciadv.aaz3173 (2020).

\section{ACKNOWLEDGEMENTS}

We thank R. MacDonald and W. Harris (Cambridge University, UK), A. Schier and F. Engert (Harvard University, USA), M. Ahrens (HHMI, Janelia Farm, USA) and K. Kawakami (SOKENDAI, The Graduate University for Advanced Studies, Japan) for transgenic lines. We thank C. Mosimann and M. Jinek for kindly providing us with Cas9 protein. We thank M. Walther, K. Dannenhauer and H. Möckel in Zürich, and S. Eggen, V. Nguyen, M. Andresen and the zebrafish facility support team in Trondheim for excellent technical and animal support. We also thank E. Brodtkorb (St. Olav's University Hospital and NTNU, Norway) and M. Gesemann for critical comments on the manuscript and the Yaksi and Neuhauss lab members for stimulating discussions. This work was funded by the Swiss National Science Foundation Grant 31003A_173083 (A.L.H., N.R., S.N., S.C.F.N), UZH Forschungskredit Candoc Grant K74417-01-01 (A.L.H.), Flanders Science Foundation (FWO) Grant (E.Y.), RCN FRIPRO Research Grant 314212 (E.Y.), RCN FRIPRO Research Grant 314189 (N.J.Y.), Medical Student's Research Programme NTNU (A.J.), and The Liaison Committee for Education, Research and Innovation in Central Norway ('Samarbeidsorganet') Grant (S.M-S., N.JY., E.Y.). Work in the E.Y. lab is funded by the Kavli Institute for Systems Neuroscience at NTNU. 
A.L.H., N.J.Y., E.Y. and S.C.F.N conceptualized the study; A.L.H., A.J., N.R., S.N. and E.A. performed experiments; A.L.H., A.J., N.R., E.A., N.J.Y. and E.Y. analysed data; A.L.H., A.J., L.L., N.J.Y. and E.Y. developed custom R and Matlab codes; A.L.H., N.J.Y. and E.Y. prepared all figures; A.L.H., N.J.Y., E.Y. and S.C.F.N wrote the manuscript; A.L.H., A.J., S.M.S., N.J.Y., E.Y. and S.C.F.N. edited the manuscript with the help of all authors; N.J.Y., E.Y. and S.C.F.N. acquired funding and supervised the students.

\section{COMPETING INTERESTS}

All authors report no conflict of interest.

\section{ADDITIONAL INFORMATION}

Correspondence and requests for materials should be addressed to N.J.Y. (email: nathalie.jurischyaksi@ntnu.no), E.Y. (email: emre.yaksi@ntnu.no) or S.C.F.N. (email: stephan.neuhauss@mls.uzh.ch) 